\title{
THE PROTECTIVE ROLE OF VITAMIN C AGAINST PHYSIOLOGICAL AND HISTOLOGICAL CHANGES IN RATS CHRONICALLY EXPOSED FOR DIAZINON AND ALUMINUM PHOSPHIDE INSECTICIDES.
}

\author{
EI Yamany EI Zawahry; Diaa Farrag Ibrahim; Walid Ali Mohammed; Mohammad \\ Fathy Abdel Naby
}

Zoology Department, Faculty of Science, Al-Azhar University, Nasr City, Cairo, Egypt.

\begin{abstract}
:
Background: Insecticides occupy a rather unique position among many chemicals that man encounters daily, in that they are deliberately added to the environment for the purpose of killing or injuring some form of life. Vitamin $\mathrm{C}$ consider as antioxidant substance which has a protective role against deleterious effects of Diazinon and Aluminium phosphide. The aim is to evaluate effects of Diazinon, Aluminum phosphide and vitamin $\mathrm{C}$ on liver, kidney and brain of albino rats. Results: data shows a significant increase in liver enzymes AST, ALT in addition to GGT and ALP by the action of the used pesticides Diazinon and Aluminum phosphide also this pesticides cause a significant increase in serum urea, uric acid and creatinine while the histological study showed that the chronic treatment with doses of the insecticides induced different deleterious histopathological lesions in the brain of treated rats. The treatment using vitamin $\mathrm{C}$ cause amelioration in liver functions tests and kidney functions tests, although, all these tests did not show any significant decrease. Conclusion: Vitamin $\mathrm{C}$ can ameliorate the deleterious effects of Diazinon and Aluminum phosphide on liver and kidney.
\end{abstract}

\section{INTRODUCTION:}

The disappearance of pesticides residues at a given location does not mean the end of the problem because they can be translocated, bioconcentrated or conversed into more dangerous chemicals (Matsumura, 1985).

Pesticides that comprise a large group substance including insecticides, fungicides, herbicides and rodenticides have been shown to produce differential and several effects on immune system in humans (Roberta, et al. 2000).

The organophosphate insecticides diazinon is widely used in agriculture or in homes and gardens (WHO, 1998).

Diazinon is an organophosphate insecticide and acaricide developed in the early 1950s. It is also used throughout the world to control public health, and is applied to control ectoparasites in veterinary medicine (Watterson, 1999). It causes changes in liver enzymes and biochemical indices and swelling of mitochondria in hepatocytes (Kalender $\boldsymbol{e t} \boldsymbol{a l}$., 2005). Also diazinon causes toxic effects on blood cells, spleen, thymus and lymph nodes of rats (Handy et al., 2002). Diazinon produced by Geigy, is an organophosphorus compound, thus active through cholinesterase inhibition, leads to the accumulation of acetylcholine at the neuromuscular junction, autonomic ganglia and parasympathetic neuroeffector junction (Hobbiger, 1964).

Pardue et al., (1970), found one of organophosphorus compounds, hydroxydiazinon as a metabolite in field sprayed kale, and as a photoalteration product of diazinon. Aluminum 
Phosphide (ALP) is a widespread cause of poisoning from industrial and agricultural chemical exposure (Gupta et al., 2003) and is accompanied with nausea, vomiting, severe shock, acute respiratory distress, altered sensorium and coma. Hypotension and tachycardia and marked bradycadia are other symptoms associated with Aluminum Phosphide poisoning (Sudakin, 2005). Acute hypoxic encephalopathy due to Aluminum Phosphide exposure has also been reported (Dua and Gill, 2004) which may lead to death as a result of complete depression of central nervous system and paralysis of the respiratory centers of the brain (WHO, 1988).

\section{MATERIAL AND METHODS:}

\section{-Experimental design and animals:}

Sixty adult male albino rats (Rattus norvegicus) of about $90 \pm 10$ gm weight were obtained from the animal Breeding House of the Research Institute of Ophthalmology, Giza, Egypt. Animals were housed in metal cages for one week, as an acclimatization period, under the laboratory conditions. The rats were fed a commercial balanced ration and allowed free to excess of water. Abnormally noticed animals were eliminated. There after, the animals were divided into ten groups in separated cages $(40 \times 60 \times 30 \mathrm{~cm})$, each group contained 6 individuals.

\section{-Experimental Groups:}

1- The Control groups (C): without any treatment or additives for the ration or water for 5 weeks and 10 weeks.

2- Diazinon groups (D): received Diazinon which only $(64 \mathrm{mg} / \mathrm{kg}$ b. w. LD50) twice weekly for the whole period of the experiment for 5 weeks and 10 weeks.

3- Diazinon + Vitamin C groups (D+C): where rats given Diazinon (64 mg/kg b. w. LD50 twice weekly) and supplemented with Vitamin C (5 mg/kg) for all the experiment period for 5 weeks and 10 weeks.

4- Aluminum Phosphide groups (A): received diazinon which only $(5.8 \mathrm{mg} / \mathrm{kg} \mathrm{b}$. w. LD50) twice weekly for the whole period of the experiment for 5 weeks and 10 weeks.

5- Aluminum Phosphide + Vitamin $\mathbf{C}$ groups $(\mathbf{D + C})$ : where rats given Diazinon (5.8 $\mathrm{mg} / \mathrm{kg} \mathrm{b}$. w. LD50 twice weekly) and supplemented with Vitamin C (5 mg/kg) for all the experiment period for 5 weeks and 10 weeks.

The experimental begins by oral administration of antioxidant (Vitamin C) twice a week followed by oral administration of Diazinon or Aluminum Phosphide insecticides (from The Agricultural Research Center, Dokki) twice a week. Concentrated ration (Diet) [which includes: $67 \%$ carbohydrates, 22\% proteins, 5\% fibers, 3\% lipids and 3\% Solids (mineral salts)] and 15-30 cm of water were ard libitum. The body weight of all groups taken twice a week. The experiment continues for 10 weeks.

At the end of ten weeks of the experiment, rats were sacrificed, samples from Brain, and Blood were collected, and serum was obtained at once, which blood sample were centrifuged at $3000 \mathrm{rpm}$, for $10 \mathrm{~min}$, by using centrifuge. Non hemolized serum was then aspirated into clean dry ependorph and stored at $-20 \mathrm{C}$ till used for biochemical analysis.

\section{Serum liver function tests:}

\section{1- Determination of Aminotransaminases activities:}

Aspartate Aminotransaminase ASAT (GOT) and Alanine Aminotransaminase ALAT (GPT) - Were estimated according to the method of Reitman and Frankel (1957) using Bio-Merieux kits. 
2- Determination of Alkaline Phosphatase (ALP) activity:

ALP Activity was estimated according to the method of Belfield and Goldberg (1971) using Bio-Merieux kits.

\section{3- Determination of Total Bilirubin concentration:}

Total bilirubin was estimated according to (Tietz, 1986a) using Randox kits.

\section{4- Determination of Gamma Glutamate Transaminase ( $\gamma$-GT):}

$\gamma$-GT is determined according to the method of Rosalki et al. (1970) using a commercial kit derived from Randox.

\section{5- Determination of Total Protein level:}

Total protein was estimated according to the method of Henry (1964) using Randox kits.

\section{6- Determination of Albumin level:}

Albumin was estimated according to the method of Doumas et al. (1971) using Boheringer kits.

\section{Serum Kidney function tests:}

1- Enzymatic Determination of Urea level:

Urea level was estimated according to the method of (Patton \& Crouch 1977) using Boheringer kits.

\section{2- Enzymatic Determination of Creatinine level:}

Creatinine level was estimated in the serum according to the method of (Henry, 1974) using Boheringer kits.

\section{3- Enzymatic Determination of Uric acid concentration:}

Uric acid concentration was estimated calorimetrically according to the method of (Fossati et al. 1980) using Randox kits.

- Statistical Analysis:

The Statistical analysis of the obtained data was done according to Armitage (1974) and Lentner $\boldsymbol{e t}$ al. (1982). The analysis was revised by Quattro Pro for windows program version (2) Microsoft Windows version (7) and graphics were drowned using Microsoft Excel (2003). The obtained data were assessed by calculation of the mean (M), Standard Deviation (SD) and percentage of change.

\section{RESULTS:}

\section{Liver function tests:}

\section{1- Aspartate Aminotransaminase activity (ASAT):}

The indicated results in table (1) show Aspartate Aminotransaminase activity (ASAT):

In 5 Weeks: The mean values of the control group (C), Diazinon (D), Diazinon + Vitamin C $(\mathrm{D}+\mathrm{C})$, Aluminum Phosphide $(\mathrm{A})$ and Aluminum Phosphide + Vitamin $\mathrm{C}(\mathrm{A}+\mathrm{C})$ were 63.2, 98.3, 77.3, 85.8, and 79.8. In Addition the percentage of change in Aspartate Aminotransaminase (ASAT) activity for the treated groups (D), (D + C), (A) and $(A+C)$ 
were significantly increased than the control as represent in the table (1) which were $55.7 \%$, $22.4 \%, 35.9 \%$ and $16.9 \%$ respectively.

In 10 Weeks: The mean values were $68.2,123.0,90.3,105.5$ and $81.5 \mathrm{mg} / \mathrm{dl}$ for the control group (C), (D), (D + C), (A) and $(A+C)$. In Addition the percentage of change in Aspartate Aminotransaminase (ASAT) activity for the treated groups (D), (D + C), (A) and $(A+C)$ were significantly increased than the control as represent in the table (1) which were $80.4 \%$, $32.5 \%, 54.8 \%$ and $19.6 \%$ respectively.

Statistical analysis: The statistical analysis for t-test shows a very high significant increase in the treated groups (D) and (A) comparing with the control group (C), in addition there is high significant increase in the treated groups $(D+C)$ and $(A+C)$ comparing with the control group (C) in 5 weeks while the statistical analysis for t-test shows a very highly significant increase in the treated groups $(\mathrm{D}),(\mathrm{D}+\mathrm{C}),(\mathrm{A})$ and $(\mathrm{A}+\mathrm{C})$ comparing with the control group (C) in 10 weeks.

\section{2- Alanine Aminotransaminase activity (ALAT):}

The obtained results in table (2) indicate Alanine Aminotransaminase (ALAT):

In 5 Weeks: The mean values of the control group (C), Diazinon (D), Diazinon + Vitamin C $(\mathrm{D}+\mathrm{C})$, Aluminum Phosphide $(\mathrm{A})$ and Aluminum Phosphide + Vitamin C $(\mathrm{A}+\mathrm{C})$ were $34.7,70.2,59.0,64.2$ and $56.0 \mathrm{mg} / \mathrm{dl}$ respectively. In Addition the percentage of change in Alanine Aminotransaminase (ALAT) activity for the treated groups (D), (D + C), (A) and (A $+\mathrm{C})$ were significantly increased than the control as represent in the table (2) which were $102.4 \%, 70.2 \%, 85.1 \%$ and $61.5 \%$ respectively.

In 10 Weeks: The mean values were $38.3,126.7,84.8,103.3$ and $78.5 \mathrm{mg} / \mathrm{dl}$ for the control group $(\mathrm{C}),(\mathrm{D}),(\mathrm{D}+\mathrm{C}),(\mathrm{A})$ and $(\mathrm{A}+\mathrm{C})$. In Addition the percentage of change in Alanine Aminotransaminase (ALAT) activity for the treated groups (D), (D + C), (A) and (A + C) were significantly increased than the control as represent in the table (2) which were 230.5 $\%, 121.3 \%, 169.6 \%$ and $104.8 \%$ respectively.

Statistical analysis: The statistical analysis for t-test shows a very high significant increase in the treated groups $(D),(D+C),(A)$ and $(A+C)$ comparing with the control group $(C)$ in 5 weeks and 10 weeks.

\section{3- Alkaline Phosphatase activity (ALP):}

The obtained results in table (3) shows alkaline phosphatase activity (ALP):

In 5 Weeks: The mean values of the control group (C), Diazinon (D), Diazinon + Vitamin C $(\mathrm{D}+\mathrm{C})$, Aluminum Phosphide $(\mathrm{A})$ and Aluminum Phosphide + Vitamin C $(\mathrm{A}+\mathrm{C})$ were $38.2,72.3,51.2,68.2$ and $47.3 \mathrm{mg} / \mathrm{dl}$ respectively. In Addition the percentage of change in Alkaline Phosphatase (ALP) activity for the treated groups (D), (D + C), (A) and (A + C) were significantly increased than the control as represent in the table (3) which were $89.5 \%$, $34.1 \%, 78.6 \%$ and $24.0 \%$ respectively.

In 10 Weeks: The mean values were 69.5, 141.2, 85.7, 126.2 and $75.7 \mathrm{mg} / \mathrm{dl}$ for the control group (C), (D), $(\mathrm{D}+\mathrm{C}),(\mathrm{A})$ and $(\mathrm{A}+\mathrm{C})$. In Addition the percentage of change in Alkaline Phosphatase (ALP) activity for the treated groups (D), $(\mathrm{D}+\mathrm{C}),(\mathrm{A})$ and $(\mathrm{A}+\mathrm{C})$ were significantly increased than the control as represent in the table (3) which were $103.1 \%$, $23.3 \%, 81.5 \%$ and $8.9 \%$ respectively.

Statistical analysis: The statistical analysis for t-test shows a very high significant increase in: the treated groups (D), (D+C) and (A) comparing with the control group (C), in addition there is significant increase when group $(C)$ compared to group $(A+C)$ in 5 weeks. the 
statistical analysis for t-test shows a very highly significant increase in the treated groups (D) and (A) comparing with the control group (C) in 10 weeks.

\section{4- $\gamma$-Glutamate Transaminase activity (GGT):}

The obtained results in table (4) shows $\gamma$-glutamate transaminase activity (GGT):

In 5 Weeks: The mean values of the control group (C), Diazinon (D), Diazinon + Vitamin C $(\mathrm{D}+\mathrm{C})$, Aluminum Phosphide $(\mathrm{A})$ and Aluminum Phosphide + Vitamin C $(\mathrm{A}+\mathrm{C})$ were 6.7, 26.2, 18.8, 23.2 and $17.3 \mathrm{mg} / \mathrm{dl}$ respectively. In Addition the difference in $\gamma$-glutamate transaminase (GGT) activity for the treated groups (D), $(\mathrm{D}+\mathrm{C}),(\mathrm{A})$ and $(\mathrm{A}+\mathrm{C})$ were significantly increased than the control as represent in the table (4) which were $290.6 \%$, $181.0 \%, 245.8 \%$ and $158.7 \%$ respectively.

In 10 Weeks: The mean values were 7.0, 29.7, 23.3, 26.2 and $20.5 \mathrm{mg} / \mathrm{dl}$ for the control group (C), (D), $(\mathrm{D}+\mathrm{C}),(\mathrm{A})$ and $(\mathrm{A}+\mathrm{C})$. In Addition the difference in $\gamma$-glutamate transaminase (GGT) activity for the treated groups (D), $(\mathrm{D}+\mathrm{C}),(\mathrm{A})$ and $(\mathrm{A}+\mathrm{C})$ were significantly increased than the control as represent in the table (4) which were $322.7 \%$, $232.3 \%, 272.8 \%$ and $192.0 \%$ respectively.

Statistical analysis: The statistical analysis for t-test shows a very high significant increase in: the treated groups $(D),(D+C),(A)$ and $(A+C)$ comparing with the control group $(C)$ in 5 weeks and 10 weeks.

\section{5- Total Bilirubin Concentration:}

The obtained results in table (5) shows total bilirubin concentration:

In 5 Weeks: The mean values of the control group (C), Diazinon (D), Diazinon + Vitamin C $(\mathrm{D}+\mathrm{C})$, Aluminum Phosphide $(\mathrm{A})$ and Aluminum Phosphide + Vitamin C $(\mathrm{A}+\mathrm{C})$ were 0.8 , 2.6, 1.8, 2.1 and $1.6 \mathrm{mg} / \mathrm{dl}$ respectively. In Addition the percentage of change in total bilirubin concentration for the treated groups $(\mathrm{D}),(\mathrm{D}+\mathrm{C}),(\mathrm{A})$ and $(\mathrm{A}+\mathrm{C})$ were significantly increased than the control as represent in the table (5) which were $226.9 \%$, $133.3 \%, 173.1 \%$ and $101.3 \%$ respectively.

In 10 Weeks: The mean values were $0.9,2.8,2.0,2.5$ and $1.8 \mathrm{mg} / \mathrm{dl}$ for the control group (C), (D), $(\mathrm{D}+\mathrm{C}),(\mathrm{A})$ and $(\mathrm{A}+\mathrm{C})$. In Addition the percentage of change in total bilirubin concentration for the treated groups $(\mathrm{D}),(\mathrm{D}+\mathrm{C}),(\mathrm{A})$ and $(\mathrm{A}+\mathrm{C})$ were significantly increased than the control as represent in the table (5) which were $215.9 \%, 125.0 \%, 178.4$ $\%$ and $108.0 \%$ respectively.

Statistical analysis: The statistical analysis for t-test shows a very high significant increase in the treated groups $(D),(D+C),(A)$ and $(A+C)$ comparing with the control group $(C)$ in 5 weeks and 10 weeks.

\section{6- Total Protein level (g/dl):}

The obtained results in table (6) shows total protein level:

In 5 Weeks: The mean values of the control group (C), Diazinon (D), Diazinon + Vitamin C $(\mathrm{D}+\mathrm{C})$, Aluminum Phosphide $(\mathrm{A})$ and Aluminum Phosphide + Vitamin C $(\mathrm{A}+\mathrm{C})$ were 6.7, 4.5, 5.1, 4.8 and $5.3 \mathrm{mg} / \mathrm{dl}$ respectively. In Addition the difference in total protein level for the treated groups $(D),(D+C),(A)$ and $(A+C)$ were significantly decreased than the control as represent in the table (6) which were $-33.0 \%,-24.4 \%,-28.3 \%$ and $-21.9 \%$ respectively.

In 10 Weeks: The mean values were $6.9,4.2,4.8,4.6$ and $5.1 \mathrm{mg} / \mathrm{dl}$ for the control group (C), (D), $(\mathrm{D}+\mathrm{C}),(\mathrm{A})$ and $(\mathrm{A}+\mathrm{C})$. In Addition the difference in total protein level for the 
treated groups $(\mathrm{D}),(\mathrm{D}+\mathrm{C}),(\mathrm{A})$ and $(\mathrm{A}+\mathrm{C})$ were significantly decreased than the control as represent in the table (6) which were $-39.1 \%,-30.7 \%,-33.2 \%$ and $-26.0 \%$ respectively.

Statistical analysis: The statistical analysis for t-test shows a very high significant decrease in: the treated groups (D), (D+C) and (A) comparing with the control group (C) in addition, there is high significant decrease when group $(C)$ compared to group $(A+C)$ in 5 weeks. The statistical analysis for t-test shows a very highly significant decrease in: the treated groups (D) and (A) comparing with the control group (C) in 10 weeks.

\section{7- Albumin Concentration (g/dl):}

The obtained results in table (7) shows albumin:

In 5 Weeks: The mean values of the control group (C), Diazinon (D), Diazinon + Vitamin C $(\mathrm{D}+\mathrm{C})$, Aluminum Phosphide (A) and Aluminum Phosphide + Vitamin C $(\mathrm{A}+\mathrm{C})$ were 4.1, 2.9, 3.4, 3.2 and $3.7 \mathrm{mg} / \mathrm{dl}$ respectively. In Addition this difference in albumin concentration for the treated groups $(D),(D+C),(A)$ and $(A+C)$ were significantly decreased than the control as represent in the table (7) which were $-30.5 \%,-16.6 \%,-22.7 \%$ and $-9.0 \%$ respectively.

In 10 Weeks: The mean values were 4.2, 2.7, 3.2, 3.0 and $3.5 \mathrm{mg} / \mathrm{dl}$ for the control group (C), (D), $(\mathrm{D}+\mathrm{C}),(\mathrm{A})$ and $(\mathrm{A}+\mathrm{C})$. In Addition this variance in albumin concentration for the treated groups $(D),(D+C),(A)$ and $(A+C)$ were significantly decreased than the control as represent in the table (7) which were $-36.2 \%,-24.4 \%,-29.8 \%$ and $-18.0 \%$ respectively.

Statistical analysis: The statistical analysis for t-test shows a very highly significant decrease in: the treated groups (D) and (A) comparing with the control group (C) in 5 weeks also, the statistical analysis for t-test shows a very highly significant decrease in the treated groups (D), (D+C), (A) and $(A+C)$ comparing with the control group (C) in 10 weeks

Table (1): The mean \pm SD show Aspartate Aminotransaminase activity (ASAT) (U/L) in albino rats protected with Vitamin $\mathrm{C}$ against chronic treated with Diazinon and Aluminum Phosphide.

\begin{tabular}{|c|c|c|c|c|c|c|}
\hline \multicolumn{2}{|l|}{$\begin{array}{l}\text { Treatment } \\
\text { (Groups) }\end{array}$} & Control & Diazinon & $\begin{array}{c}\text { Diazinon } \\
+ \\
\text { Vitamin C }\end{array}$ & $\begin{array}{l}\text { Aluminum } \\
\text { Phosphide }\end{array}$ & $\begin{array}{c}\text { Aluminum } \\
\text { Phosphide } \\
+ \\
\text { Vitamin C }\end{array}$ \\
\hline \multirow{2}{*}{$\begin{array}{c}5 \\
\text { Weeks }\end{array}$} & $\begin{array}{l}\text { Mean } \\
\pm \text { SD }\end{array}$ & $\begin{array}{r}63.17 \\
\pm 5.23\end{array}$ & $\begin{array}{c}98.33 * * * \\
\pm 15.19\end{array}$ & $\begin{array}{c}77.33 \text { ** } \\
\pm 8.12\end{array}$ & $\begin{array}{c}85.83 * * * \\
\pm 7.88\end{array}$ & $\begin{array}{c}73.83 * * \\
\pm 6.27\end{array}$ \\
\hline & \% of Change & & $\uparrow 55.7$ & $\uparrow 22.4$ & $\uparrow 35.9$ & $\uparrow 16.9$ \\
\hline \multirow{2}{*}{$\begin{array}{c}10 \\
\text { Weeks }\end{array}$} & $\begin{array}{l}\text { Mean } \\
\pm \text { SD }\end{array}$ & $\begin{array}{r}68.17 \\
\pm 5.19\end{array}$ & $\begin{array}{c}123.00 * * * \\
\pm 17.74\end{array}$ & $\begin{array}{c}90.33 * * * \\
\quad \pm 7.84\end{array}$ & $\begin{array}{c}105.50 * * * \\
\pm 7.01\end{array}$ & $\begin{array}{c}81.50 * * * \\
\pm 6.25\end{array}$ \\
\hline & \% of Change & & $\uparrow 80.4$ & $\uparrow 32.5$ & $\uparrow 54.8$ & $\uparrow 19.6$ \\
\hline
\end{tabular}


Table (2): The mean \pm SD show Alanine Aminotransaminase (ALAT) (U/L) in albino rats protected with Vitamin $\mathrm{C}$ against chronic treated with Diazinon and Aluminum Phosphide.

\begin{tabular}{|c|c|c|c|c|c|c|}
\hline Weeks & $\begin{array}{r}\begin{array}{c}\text { Treatment } \\
\text { (Groups) }\end{array} \\
\end{array}$ & Control & Diazinon & $\begin{array}{c}\text { Diazinon } \\
\stackrel{+}{ } \\
\text { Vitamin } \mathbf{C}\end{array}$ & $\begin{array}{l}\text { Aluminum } \\
\text { Phosphide }\end{array}$ & $\begin{array}{c}\text { Aluminum } \\
\text { Phosphide } \\
+ \\
\text { Vitamin C }\end{array}$ \\
\hline \multirow{2}{*}{$\begin{array}{c}5 \\
\text { Weeks }\end{array}$} & $\begin{array}{l}\text { Mean } \\
\pm \text { SD }\end{array}$ & $\begin{array}{c}34.67 \\
\pm 3.39\end{array}$ & $\begin{array}{c}70.17 * * * \\
\pm 4.31\end{array}$ & $\begin{array}{c}59.00 * * * \\
\pm 7.21\end{array}$ & $\begin{array}{c}64.17 * * * \\
\pm 6.11\end{array}$ & $\begin{array}{c}56.00 * * * \\
\pm 6.10\end{array}$ \\
\hline & $\%$ of Change & & $102.4 \uparrow$ & $70.2 \uparrow$ & $85.1 \uparrow$ & $61.5 \uparrow$ \\
\hline \multirow{2}{*}{$\begin{array}{c}10 \\
\text { Weeks }\end{array}$} & $\begin{array}{l}\text { Mean } \\
\pm \text { SD }\end{array}$ & $\begin{array}{r}38.33 \\
\pm 2.16\end{array}$ & $\begin{array}{c}126.67 * * * \\
\quad \pm 19.12\end{array}$ & $\begin{array}{c}84.83 * * * \\
\pm 6.85\end{array}$ & $\begin{array}{c}103.33 * * * \\
\pm 13.75\end{array}$ & $\begin{array}{c}78.50 * * * \\
\pm \mathbf{5 . 4 3}\end{array}$ \\
\hline & $\%$ of Change & & $230.5 \uparrow$ & $121.3 \uparrow$ & $169.6 \uparrow$ & $104.8 \uparrow$ \\
\hline
\end{tabular}

Table (3): The mean \pm SD show Alkaline Phosphatase (ALP) activity (U/L) in albino rats protected with Vitamin $\mathrm{C}$ against chronic treated with Diazinon and Aluminum Phosphide.

\begin{tabular}{|c|c|c|c|c|c|c|}
\hline Weeks & $\begin{array}{c}\text { Treatment } \\
\text { (Groups) }\end{array}$ & Control & Diazinon & $\begin{array}{c}\text { Diazinon } \\
\stackrel{+}{\text { Vitamin } \mathrm{C}}\end{array}$ & $\begin{array}{l}\text { Aluminum } \\
\text { Phosphide }\end{array}$ & $\begin{array}{c}\text { Aluminum } \\
\text { Phosphide } \\
+ \\
\text { Vitamin C }\end{array}$ \\
\hline \multirow{2}{*}{$\begin{array}{c}5 \\
\text { Weeks }\end{array}$} & $\begin{array}{l}\text { Mean } \\
\pm \text { SD }\end{array}$ & $\begin{array}{c}38.17 \\
\pm 3.66\end{array}$ & $\begin{array}{c}72.33 * * * \\
\pm \mathbf{5 . 3 9}\end{array}$ & $\begin{array}{c}51.17 * * * \\
\pm 3.87\end{array}$ & $\begin{array}{c}68.17 * * * \\
\pm 5.74\end{array}$ & $\begin{array}{l}47.33 * \\
\pm 7.53\end{array}$ \\
\hline & $\%$ of Change & & $89.5 \uparrow$ & $34 . .1 \uparrow$ & $78.6 \uparrow$ & $24.0 \uparrow$ \\
\hline \multirow{2}{*}{$\begin{array}{c}10 \\
\text { Weeks }\end{array}$} & $\begin{array}{l}\text { Mean } \\
\pm \text { SD }\end{array}$ & $\begin{array}{c}69.50 \\
\pm 10.67\end{array}$ & $\begin{array}{c}141.17 * * * \\
\pm 25.40\end{array}$ & $\begin{array}{l}85.67 \mathrm{~ns} \\
\pm 11.29\end{array}$ & $\begin{array}{c}126.17 * * * \\
\pm 13.41\end{array}$ & $\begin{array}{l}75.67 \mathrm{~ns} \\
\pm \mathbf{1 2 . 3 6}\end{array}$ \\
\hline & $\%$ of Change & & $103.1 \uparrow$ & $23.3 \uparrow$ & $81.5 \uparrow$ & $8.9 \uparrow$ \\
\hline
\end{tabular}

Table (4): The mean \pm SD show $\gamma$-Glutamate Transaminase activity (GGT) (U/L) in albino rats Protected with Vitamin $\mathrm{C}$ against treated with Diazinon and Aluminum Phosphide toxicity.

\begin{tabular}{|c|c|c|c|c|c|c|}
\hline Weeks & $\begin{array}{r}\begin{array}{c}\text { Treatment } \\
\text { (Groups) }\end{array} \\
\end{array}$ & Control & Diazinon & $\begin{array}{c}\text { Diazinon } \\
+ \\
\text { Vitamin C }\end{array}$ & $\begin{array}{l}\text { Aluminum } \\
\text { Phosphide }\end{array}$ & $\begin{array}{c}\text { Aluminum } \\
\text { Phosphide } \\
+ \\
\text { Vitamin C } \\
\end{array}$ \\
\hline \multirow{2}{*}{$\begin{array}{c}5 \\
\text { Weeks }\end{array}$} & $\begin{array}{l}\text { Mean } \\
\pm \text { SD }\end{array}$ & $\begin{array}{c}6.70 \\
\pm 0.53\end{array}$ & $\begin{array}{c}26.17 * * * \\
\quad \pm 4.92\end{array}$ & $\begin{array}{c}18.83 * * * \\
\pm 5.91\end{array}$ & $\begin{array}{c}23.17 * * * \\
\quad \pm 3.76\end{array}$ & $\begin{array}{c}17.33 * * * \\
\pm 3.27\end{array}$ \\
\hline & $\%$ of Change & & $290.6 \uparrow$ & $181.0 \uparrow$ & $245.8 \uparrow$ & $158.7 \uparrow$ \\
\hline \multirow{2}{*}{$\begin{array}{c}10 \\
\text { Weeks }\end{array}$} & $\begin{array}{l}\text { Mean } \\
\pm \text { SD }\end{array}$ & $\begin{array}{c}7.02 \\
\pm 0.25\end{array}$ & $\begin{array}{c}29.67 * * * \\
\pm 5.13\end{array}$ & $\begin{array}{c}23.33 * * * \\
\pm 5.01\end{array}$ & $\begin{array}{c}26.17 * * * \\
\pm 4.92\end{array}$ & $\begin{array}{c}20.50 * * * \\
\pm 3.27\end{array}$ \\
\hline & $\%$ of Change & & $322.7 \uparrow$ & $232.3 \uparrow$ & $272.8 \uparrow$ & $192.0 \uparrow$ \\
\hline
\end{tabular}


Table (5): The mean \pm SD show Total Bilirubin concentration $(\mathrm{mg} / \mathrm{dl})$ in albino rats protected with Vitamin $\mathrm{C}$ against chronic treated with Diazinon and Aluminum Phosphide.

\begin{tabular}{|c|c|c|c|c|c|c|}
\hline Weeks & $\begin{array}{r}\begin{array}{c}\text { Treatment } \\
\text { (Groups) }\end{array} \\
\end{array}$ & Control & Diazinon & $\begin{array}{c}\text { Diazinon } \\
+ \\
\text { Vitamin C }\end{array}$ & $\begin{array}{l}\text { Aluminum } \\
\text { Phosphide }\end{array}$ & $\begin{array}{c}\text { Aluminum } \\
\text { Phosphide } \\
+ \\
\text { Vitamin C }\end{array}$ \\
\hline \multirow{2}{*}{$\begin{array}{c}5 \\
\text { Weeks }\end{array}$} & $\begin{array}{l}\text { Mean } \\
\pm \text { SD }\end{array}$ & $\begin{array}{c}0.78 \\
\pm 0.12\end{array}$ & $\begin{array}{c}2.55 * * * \\
\pm 0.37\end{array}$ & $\begin{array}{c}1.82 * * * \\
\pm 0.37\end{array}$ & $\begin{array}{c}2.13 * * * \\
\pm 0.33\end{array}$ & $\begin{array}{c}1.57 * * * \\
\pm 0.37\end{array}$ \\
\hline & $\%$ of Change & & $226.9 \uparrow$ & $133.3 \uparrow$ & $173.1 \uparrow$ & $101.3 \uparrow$ \\
\hline \multirow{2}{*}{$\begin{array}{c}10 \\
\text { Weeks }\end{array}$} & $\begin{array}{l}\text { Mean } \\
\pm \text { SD }\end{array}$ & $\begin{array}{c}0.88 \\
\pm 0.15\end{array}$ & $\begin{array}{c}2.78 * * * \\
\pm 0.16\end{array}$ & $\begin{array}{c}1.98 * * * \\
\pm 0.31\end{array}$ & $\begin{array}{c}2.45 * * * \\
\pm 0.24\end{array}$ & $\begin{array}{c}1.83 * * * \\
\pm 0.35\end{array}$ \\
\hline & $\%$ of Change & & $215.9 \uparrow$ & $125.0 \uparrow$ & $178.4 \uparrow$ & $108.0 \uparrow$ \\
\hline
\end{tabular}

Table (6): The mean \pm SD show Total Protein concentration $(\mathrm{g} / \mathrm{dl})$ in albino rats protected with Vitamin C against chronic treated with Diazinon and Aluminum Phosphide.

\begin{tabular}{|c|c|c|c|c|c|c|}
\hline Weeks & $\begin{array}{c}\text { Treatment } \\
\text { (Groups) }\end{array}$ & Control & Diazinon & $\begin{array}{c}\text { Diazinon } \\
+ \\
\text { Vitamin } \mathrm{C}\end{array}$ & $\begin{array}{l}\text { Aluminum } \\
\text { Phosphide }\end{array}$ & $\begin{array}{c}\text { Aluminum } \\
\text { Phosphide } \\
+ \\
\text { Vitamin C }\end{array}$ \\
\hline \multirow{2}{*}{$\begin{array}{c}5 \\
\text { Weeks }\end{array}$} & $\begin{array}{l}\text { Mean } \\
\pm \text { SD }\end{array}$ & $\begin{array}{c}6.72 \\
\pm 0.23\end{array}$ & $\begin{array}{c}4.50 * * * \\
\pm 0.47\end{array}$ & $\begin{array}{c}5.08 * * * \\
\pm 0.79\end{array}$ & $\begin{array}{c}4.82 * * * \\
\pm 0.45\end{array}$ & $\begin{array}{c}5.25 * * \\
\pm 0.83\end{array}$ \\
\hline & $\%$ of Change & & $-33.0 \downarrow$ & $-24.4 \downarrow$ & $-28.3 \downarrow$ & $-21.9 \downarrow$ \\
\hline \multirow{2}{*}{$\begin{array}{c}10 \\
\text { Weeks }\end{array}$} & $\begin{array}{l}\text { Mean } \\
\pm \text { SD }\end{array}$ & $\begin{array}{c}6.93 \\
\pm 0.24\end{array}$ & $\begin{array}{c}4.22 * * * \\
\pm 0.35\end{array}$ & $\begin{array}{l}4.80 \mathrm{~ns} \\
\pm 0.42\end{array}$ & $\begin{array}{c}4.63 * * * \\
\pm 0.29\end{array}$ & $\begin{array}{l}5.13 \mathrm{~ns} \\
\pm 0.66\end{array}$ \\
\hline & $\%$ of Change & & $-39.1 \downarrow$ & $-30.7 \downarrow$ & $-33.2 \downarrow$ & $-26.0 \downarrow$ \\
\hline
\end{tabular}

Table (7): The mean \pm SD show Albumin concentration $(\mathrm{g} / \mathrm{dl})$ in albino rats protected with Vitamin $\mathrm{C}$ against chronic treated with Diazinon and Aluminum Phosphide.

\begin{tabular}{|c|c|c|c|c|c|c|}
\hline Weeks & $\begin{array}{r}\begin{array}{r}\text { Treatment } \\
\text { (Groups) }\end{array} \\
\end{array}$ & Control & Diazinon & $\begin{array}{c}\text { Diazinon } \\
+ \\
\text { Vitamin C }\end{array}$ & $\begin{array}{l}\text { Aluminum } \\
\text { Phosphide }\end{array}$ & $\begin{array}{c}\text { Aluminum } \\
\text { Phosphide } \\
+ \\
\text { Vitamin C }\end{array}$ \\
\hline \multirow{2}{*}{$\begin{array}{c}5 \\
\text { Weeks }\end{array}$} & $\begin{array}{l}\text { Mean } \\
\pm \text { SD }\end{array}$ & $\begin{array}{c}4.10 \\
\pm 0.29\end{array}$ & $\begin{array}{c}2.85 * * * \\
\pm 0.33\end{array}$ & $\begin{array}{c}3.42 \mathrm{~ns} \\
\pm 1.11\end{array}$ & $\begin{array}{c}3.17 * * * \\
\pm 0.52\end{array}$ & $\begin{array}{l}3.73 \mathrm{~ns} \\
\pm 0.67\end{array}$ \\
\hline & $\%$ of Change & & $-30.5 \downarrow$ & $-16.6 \downarrow$ & $-22.7 \downarrow$ & $-9.0 \downarrow$ \\
\hline \multirow{2}{*}{$\begin{array}{c}10 \\
\text { Weeks }\end{array}$} & $\begin{array}{l}\text { Mean } \\
\pm \text { SD }\end{array}$ & $\begin{array}{c}4.23 \\
\pm 0.33\end{array}$ & $\begin{array}{c}2.70 * * * \\
\pm 0.42\end{array}$ & $\begin{array}{l}3.20 * * * \\
\pm 0.70\end{array}$ & $\begin{array}{c}2.97 * * * \\
\pm 0.40\end{array}$ & $\begin{array}{c}3.47 * * * \\
\pm 0.47\end{array}$ \\
\hline & $\%$ of Change & & $-36.2 \downarrow$ & $-24.4 \downarrow$ & $-29.8 \downarrow$ & $-18.0 \downarrow$ \\
\hline
\end{tabular}

\section{Where:}

C $=$ Control,

$\mathbf{D}=$ Diazinon,

$\mathbf{D}+\mathbf{C}=$ Diazinon + Vitamin $\mathrm{C}$

A = Aluminum Phosphide,

SD $=$ Standard Division,

$\mathbf{A}+\mathbf{C}=$ Aluminum Phosphide + Vitamin $\mathbf{C}$

$\mathbf{n s}=$ Non Significant,

$\%$ Change $=$ Percentage of change from control,

$* *=$ High Significant.

$*$ = Significant,

$* * *=$ Very High Significant. 


\section{Kidney functions tests:}

\section{1- Urea Concentration (mg/dl):}

The obtained results in table (8) shows urea concentration:

In 5 Weeks: The mean values of the control group (C), Diazinon (D), Diazinon + Vitamin C $(\mathrm{D}+\mathrm{C})$, Aluminum Phosphide $(\mathrm{A})$ and Aluminum Phosphide + Vitamin C $(\mathrm{A}+\mathrm{C})$ were 35.7, 62.8, 52.0, 56.8 and $48.7 \mathrm{mg} / \mathrm{dl}$ respectively. In Addition this difference in urea concentration for the treated groups (D), $(\mathrm{D}+\mathrm{C}),(\mathrm{A})$ and $(\mathrm{A}+\mathrm{C})$ were significantly increased than the control as represent in the table (8) which were $76.1 \%, 45.8 \%, 59.3 \%$ and $36.5 \%$ respectively.

In 10 Weeks: The mean values were 37.3, 81.0, 65.2, 71.5 and $60.7 \mathrm{mg} / \mathrm{dl}$ for the control group $(\mathrm{C}),(\mathrm{D}),(\mathrm{D}+\mathrm{C}),(\mathrm{A})$ and $(\mathrm{A}+\mathrm{C})$. In Addition this difference in urea concentration for the treated groups $(D),(D+C),(A)$ and $(A+C)$ were significantly increased than the control as represented in the table (8) which were $117.0 \%, 74.6 \%, 91.5 \%$ and $62.5 \%$ respectively.

Statistical analysis: The statistical analysis for t-test shows a very high significant increase in: the treated groups $(D),(D+C),(A)$ and $(A+C)$ comparing with the control group $(C)$ in 5 weeks and 10 weeks.

\section{2- Creatinine Concentration ( $\mathrm{mg} / \mathrm{dl})$ :}

The obtained results in table (9) shows serum creatinine concentration:

In 5 Weeks: The mean values of the control group (C), Diazinon (D), Diazinon + Vitamin C $(\mathrm{D}+\mathrm{C})$, Aluminum Phosphide $(\mathrm{A})$ and Aluminum Phosphide + Vitamin C $(\mathrm{A}+\mathrm{C})$ were 0.8 , $1.8,1.3,1.4$ and $1.0 \mathrm{mg} / \mathrm{dl}$ respectively. In Addition this variance in creatinine concentration for the treated groups $(D),(D+C),(A)$ and $(A+C)$ were significantly increased than the control as represent in the table (9) which were $113.3 \%, 50.6 \%, 65.1 \%$ and $24.1 \%$ respectively.

In 10 Weeks: The mean values were $1.0,2.2,1.5,1.7$ and $1.3 \mathrm{mg} / \mathrm{dl}$ for the control group (C), (D), $(\mathrm{D}+\mathrm{C}),(\mathrm{A})$ and $(\mathrm{A}+\mathrm{C})$. In Addition this variance in creatinine concentration for the treated groups $(D),(D+C),(A)$ and $(A+C)$ were significantly increased than the control as represent in the table (9) which were $123.0 \%, 47.0 \%, 67.0 \%$ and $25.0 \%$ respectively.

Statistical analysis: The statistical analysis for t-test shows a very highly significant increase in the treated group (D) comparing with the control group (C), in addition there is high significant increase when group (A) compared to group (C), furthermore there is significant increase when group $(C)$ compared to groups $(D+C)$ and $(A+C)$ in 5 weeks while the statistical data for t-test Shows a very highly significant increase in the treated groups (D), (D+C) and (A) comparing with the control group (C), in addition there is high significant increase when compared between group $(C)$ and $(A+C)$ in 10 weeks.

\section{3- Uric Acid Concentration (mg/dl):}

The obtained results in table (10) shows serum uric acid concentration:

In 5 Weeks: The mean values of the control group (C), Diazinon (D), Diazinon + Vitamin C $(\mathrm{D}+\mathrm{C})$, Aluminum Phosphide $(\mathrm{A})$ and Aluminum Phosphide + Vitamin C $(\mathrm{A}+\mathrm{C})$ were 4.7, 7.6, 5.5, 6.9 and $5.1 \mathrm{mg} / \mathrm{dl}$ respectively. In Addition this variance in uric acid concentration for the treated groups $(D),(D+C),(A)$ and $(A+C)$ were significantly increased than the control as represent in the table (10) which were $60.4 \%, 16.1 \%, 45.6 \%$ and $8.5 \%$ respectively. 
In 10 Weeks: The mean values were 5.3, 12.4, 10.3, 11.2 and $8.8 \mathrm{mg} / \mathrm{dl}$ for the control group $(C),(D),(D+C),(A)$ and $(A+C)$. In Addition this variance in uric acid concentration for the treated groups $(D),(D+C),(A)$ and $(A+C)$ were significantly increased than the control as represent in the table (10) which were $134.3 \%, 93.8 \%, 110.4 \%$ and $66.4 \%$ respectively.

Statistical analysis: The statistical analysis for t-test shows a very highly significant increase in: the treated groups (D) and (A) comparing with the control group (C) in 5 weeks, also the statistical analysis for t-test shows a very highly significant increase in: the treated groups (D) and (A) comparing with the control group (C), in addition there is high significant increase in the treated groups $(\mathrm{D}+\mathrm{C})$ and $(\mathrm{A}+\mathrm{C})$ comparing with the control group (C) in 10 weeks.

Table (8): The mean \pm SD show Urea $(\mathrm{mg} / \mathrm{dl})$ in albino rats protected with Vitamin $\mathrm{C}$ against chronic treated with Diazinon and Aluminum Phosphide.

\begin{tabular}{|c|c|c|c|c|c|c|}
\hline Weeks & $\begin{array}{c}\text { Treatment } \\
\text { (Groups) }\end{array}$ & Control & Diazinon & $\begin{array}{c}\text { Diazinon } \\
+ \\
\text { Vitamin C }\end{array}$ & $\begin{array}{l}\text { Aluminum } \\
\text { Phosphide }\end{array}$ & $\begin{array}{c}\text { Aluminum } \\
\text { Phosphide } \\
+ \\
\text { Vitamin C }\end{array}$ \\
\hline \multirow{2}{*}{$\begin{array}{c}5 \\
\text { Weeks }\end{array}$} & $\begin{array}{l}\text { Mean } \\
\pm \text { SD }\end{array}$ & $\begin{array}{l}35.67 \\
\pm 4.93\end{array}$ & $\begin{array}{c}62.83 * * * \\
\pm 5.00\end{array}$ & $\begin{array}{c}52.00 * * * \\
\pm 6.48\end{array}$ & $\begin{array}{c}56.83 * * * \\
\pm 6.08\end{array}$ & $\begin{array}{c}48.67 * * * \\
\pm 2.34\end{array}$ \\
\hline & $\%$ of Change & & $76.1 \uparrow$ & $45.8 \uparrow$ & $59.3 \uparrow$ & $36.5 \uparrow$ \\
\hline \multirow{2}{*}{$\begin{array}{c}10 \\
\text { Weeks }\end{array}$} & $\begin{array}{l}\text { Mean } \\
\pm \text { SD }\end{array}$ & $\begin{array}{r}37.33 \\
\pm \mathbf{5 . 0 5}\end{array}$ & $\begin{array}{l}81.00 * * * \\
\pm 4.90\end{array}$ & $\begin{array}{c}65.17 * * * \\
\pm 6.24\end{array}$ & $\begin{array}{c}71.50 * * * \\
\pm 5.72\end{array}$ & $\begin{array}{c}60.67 * * * \\
\quad \pm 4.55\end{array}$ \\
\hline & $\%$ of Change & & $117.0 \uparrow$ & $74.6 \uparrow$ & $91.5 \uparrow$ & $62.5 \uparrow$ \\
\hline
\end{tabular}

Table (9): The mean \pm SD show Creatinine (mg/dl) in albino rats protected with Vitamin C against chronic treated with Diazinon and Aluminum Phosphide.

\begin{tabular}{|c|c|c|c|c|c|c|}
\hline Weeks & $\begin{array}{r}\text { Treatment } \\
\text { (Groups) }\end{array}$ & Control & Diazinon & $\begin{array}{c}\text { Diazinon } \\
+ \\
\text { Vitamin C }\end{array}$ & $\begin{array}{l}\text { Aluminum } \\
\text { Phosphide }\end{array}$ & $\begin{array}{c}\text { Aluminum } \\
\text { Phosphide } \\
+ \\
\text { Vitamin C }\end{array}$ \\
\hline \multirow{2}{*}{$\begin{array}{c}5 \\
\text { Weeks }\end{array}$} & $\begin{array}{l}\text { Mean } \\
\pm \text { SD }\end{array}$ & $\begin{array}{c}0.83 \\
\pm 0.15\end{array}$ & $\begin{array}{c}1.77 * * * \\
\pm 0.18\end{array}$ & $\begin{array}{l}1.25 * \\
\pm 0.31\end{array}$ & $\begin{array}{l}1.37 * * \\
\pm 0.24\end{array}$ & $\begin{array}{l}1.03 * \\
\pm 0.12\end{array}$ \\
\hline & $\%$ of Change & & $113.3 \uparrow$ & $50.6 \uparrow$ & $65.1 \uparrow$ & $24.1 \uparrow$ \\
\hline \multirow{2}{*}{$\begin{array}{c}10 \\
\text { Weeks }\end{array}$} & $\begin{array}{l}\text { Mean } \\
\pm \text { SD }\end{array}$ & $\begin{array}{c}1.0 \\
\pm 0.22\end{array}$ & $\begin{array}{c}2.23 * * * \\
\pm 0.29\end{array}$ & $\begin{array}{c}1.47 * * * \\
\pm 0.22\end{array}$ & $\begin{array}{c}1.67 * * * \\
\pm 0.20\end{array}$ & $\begin{array}{c}1.25 * * \\
\pm 0.16\end{array}$ \\
\hline & $\%$ of Change & & $123.0 \uparrow$ & $47.0 \uparrow$ & $67.0 \uparrow$ & $25.0 \uparrow$ \\
\hline
\end{tabular}


Table (10): The mean \pm SD show Uric Acid (mg/dl) in albino rats protected with Vitamin C against chronic treated with Diazinon and Aluminum Phosphide.

\begin{tabular}{|c|c|c|c|c|c|c|}
\hline Weeks & $\begin{array}{r}\text { Treatment } \\
\text { (Groups) }\end{array}$ & Control & Diazinon & $\begin{array}{c}\text { Diazinon } \\
+ \\
\text { Vitamin C }\end{array}$ & $\begin{array}{l}\text { Aluminum } \\
\text { Phosphide }\end{array}$ & $\begin{array}{c}\text { Aluminum } \\
\text { Phosphide } \\
+ \\
\text { Vitamin C } \\
\end{array}$ \\
\hline \multirow{2}{*}{$\begin{array}{c}5 \\
\text { Weeks }\end{array}$} & $\begin{array}{l}\text { Mean } \\
\pm \text { SD }\end{array}$ & $\begin{array}{c}4.72 \\
\pm 0.41\end{array}$ & $\begin{array}{c}7.57 * * * \\
\pm 0.62\end{array}$ & $\begin{array}{l}5.48 \mathrm{~ns} \\
\pm 0.87\end{array}$ & $\begin{array}{c}6.87 * * * \\
\pm 0.76\end{array}$ & $\begin{array}{l}5.12 \mathrm{~ns} \\
\pm 0.84\end{array}$ \\
\hline & $\%$ of Change & & $60.4 \uparrow$ & $16.1 \uparrow$ & $45.6 \uparrow$ & $8.5 \uparrow$ \\
\hline \multirow{2}{*}{$\begin{array}{c}10 \\
\text { Weeks }\end{array}$} & $\begin{array}{l}\text { Mean } \\
\pm \text { SD }\end{array}$ & $\begin{array}{c}5.30 \\
\pm 0.37\end{array}$ & $\begin{array}{c}12.42 * * * \\
\pm 0.87\end{array}$ & $\begin{array}{c}10.27 * * \\
\pm 0.97\end{array}$ & $\begin{array}{c}11.15 * * * \\
\pm 1.01\end{array}$ & $\begin{array}{c}8.82 * * \\
\pm 1.38\end{array}$ \\
\hline & $\%$ of Change & & $134.3 \uparrow$ & $93.8 \uparrow$ & $110.4 \uparrow$ & $66.4 \uparrow$ \\
\hline
\end{tabular}

\section{Where:}

$\mathbf{C}=$ Control,

$\mathbf{D}=$ Diazinon,

A = Aluminum Phosphide,

SD $=$ Standard Division,

ns = Non Significant,

$* *$ = High Significant.

D $+\mathbf{C}=$ Diazinon + Vitamin C

$\mathbf{A}+\mathbf{C}=$ Aluminum Phosphide + Vitamin $\mathbf{C}$

\% Change $=$ Percentage of change from control,

$*$ = Significant,

*** = Very High Significant. 


\section{Histological and Histopathological Studies:}

\section{A- The Brain:}

\section{1- Control Group :}

Microscopic examination of sections from the cerebral cortex of the control rats showed the normal histology of the layer of the cerebral cortex, the Photomicrography sections of control cerebral cortex of rats shows: $(\mathrm{P})$ pia- matter layer, $(\mathrm{Mo})$ molecular layer, (Eg) external granular, (Ep) external pyramidal, (Ig) internal granular, (Ip) internal pyramidal, (Pm) polymorphic layer and (Pc) Parkinson cells, can also see as in the (Plate 1). (Hx\&E x 100 and $\mathrm{x} 400)$.
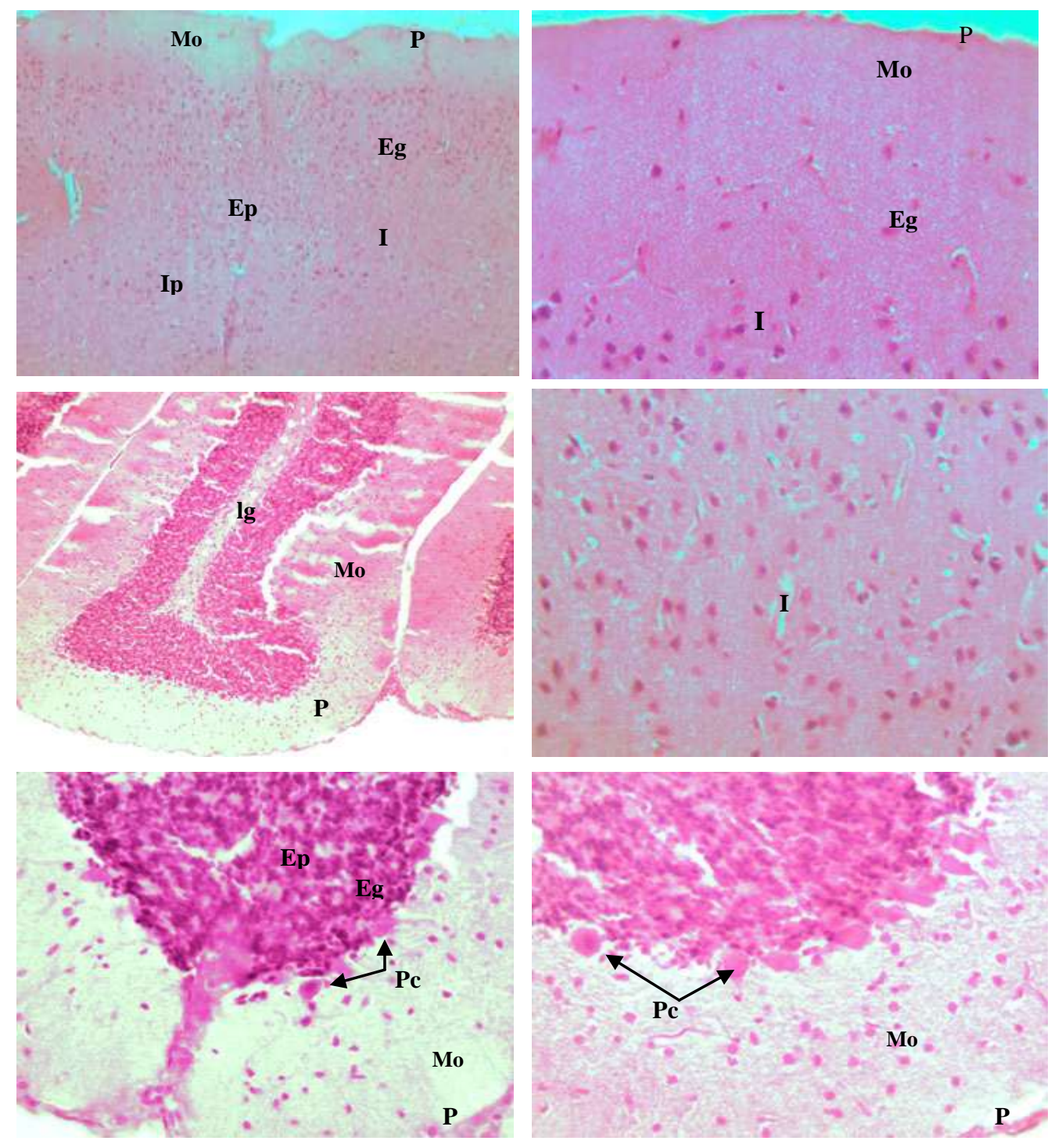

\section{Plate (1) Control Group}

Brain Sections of control Albino rats at 5 and 10 weeks. 


\section{2- Diazinon Group (5 Weeks):}

Photomicrography sections of treated rats with Diazinon in 5 weeks showing: malformed pia mater $(\mathrm{P})$ with infiltrated inflammatory cells layer (Iic), dilated and congested blood vessels (DBV) and (CBV) in pia matter. Numerous polymorphic nerve cells (Poc) and decreased number Parkinson cells (Pc) can also see as in the (Plate 2). (Hx\&E x 100 and $\mathrm{x} 400)$.
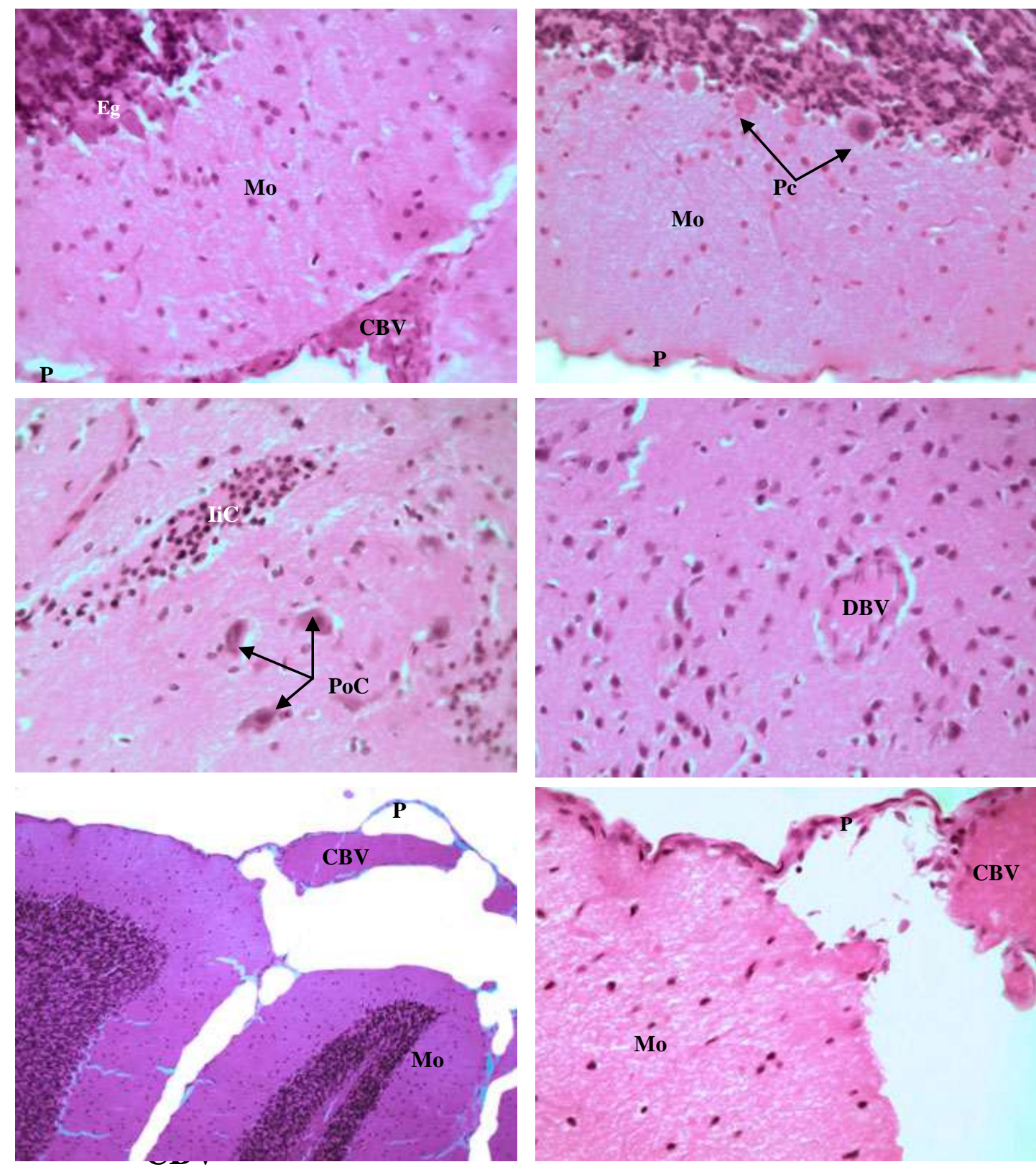

Plate (2) Diazinon Group (5 Weeks)

Brain Sections of Albino rats treated with Diazinon at 5 weeks. 


\section{3- Aluminum Phosphide Group (5 Weeks):}

Photomicrography sections of treated rats with Aluminum Phosphide in 5 weeks showing: many histopathological alteration represented by shredding of pia mater layer $(\mathrm{P})$, with infiltrated inflammatory cells layer (Iic), congested and dilated blood vessels (CBV) \& (DBV), hemorrhage $(\mathrm{H})$, necrotic areas $(\mathrm{Na})$ and also decreased number of Parkinson cells (Pc) were seen as in the (Plate 3) (Hx\&E x100 and x400).
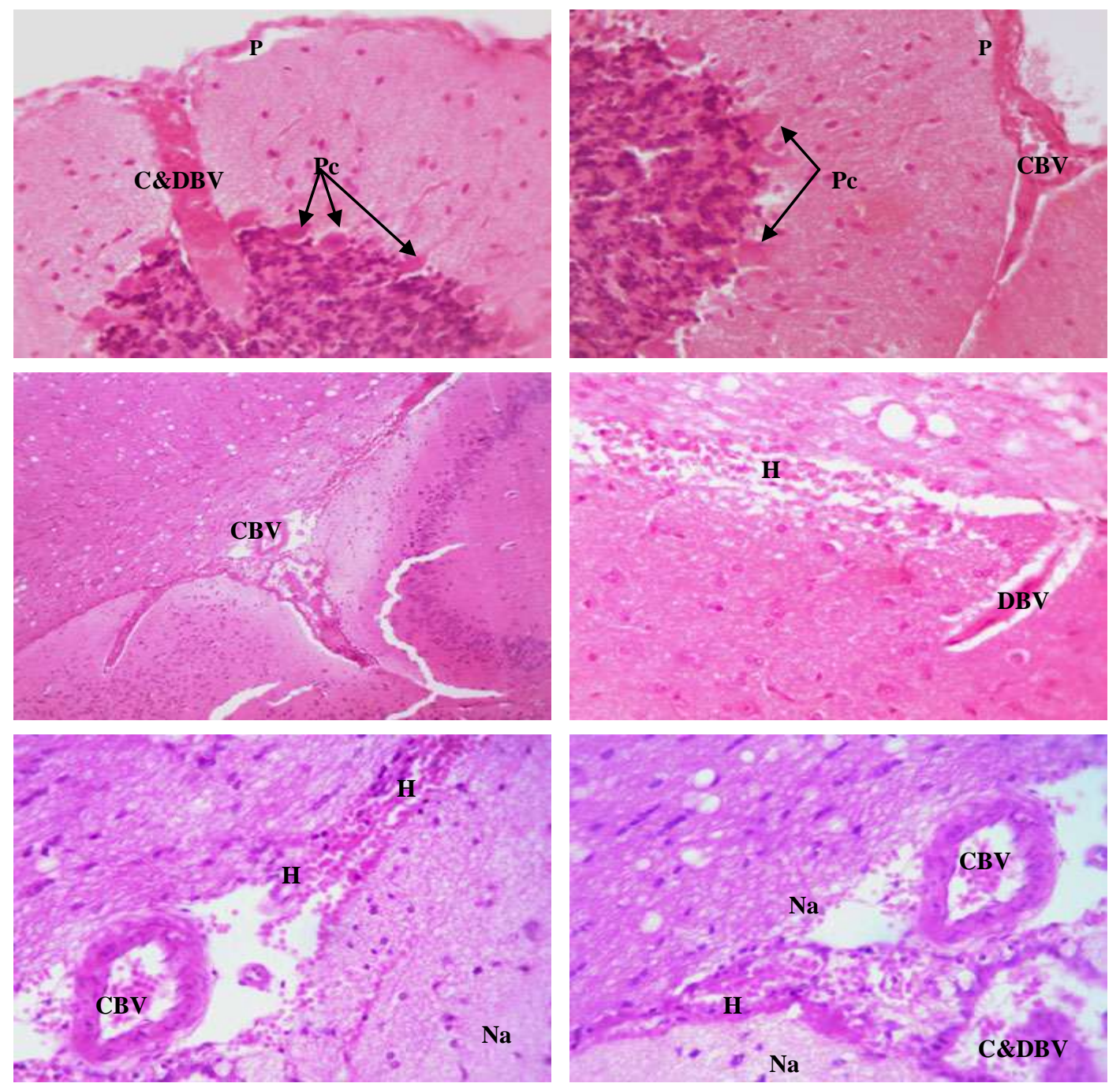

Plate (3) Aluminum Phosphide Group (5 Weeks)

Brain Sections of Albino rats treated with Aluminum Phosphide at 5 weeks. 


\section{4- $\quad$ Aluminum Phosphide Group (10 Weeks):}

Photomicrography sections revealed dilated and congested blood vessels (DBV) \& $(\mathrm{CBV})$ with fibrosis epithelial layer $(\mathrm{Fi})$ in pia- matter $(\mathrm{P})$ and loss of density in plexus nerve fibers resulted to reduce nerve cells and also carcinogenic cells (Cc) were seen forming a bulk of dark stained cells, hemorrhage $(\mathrm{H})$ and necrotic areas $(\mathrm{Na})$ also can be seen in many areas (Plate 4) (Hx\&Ex100 and x 400).
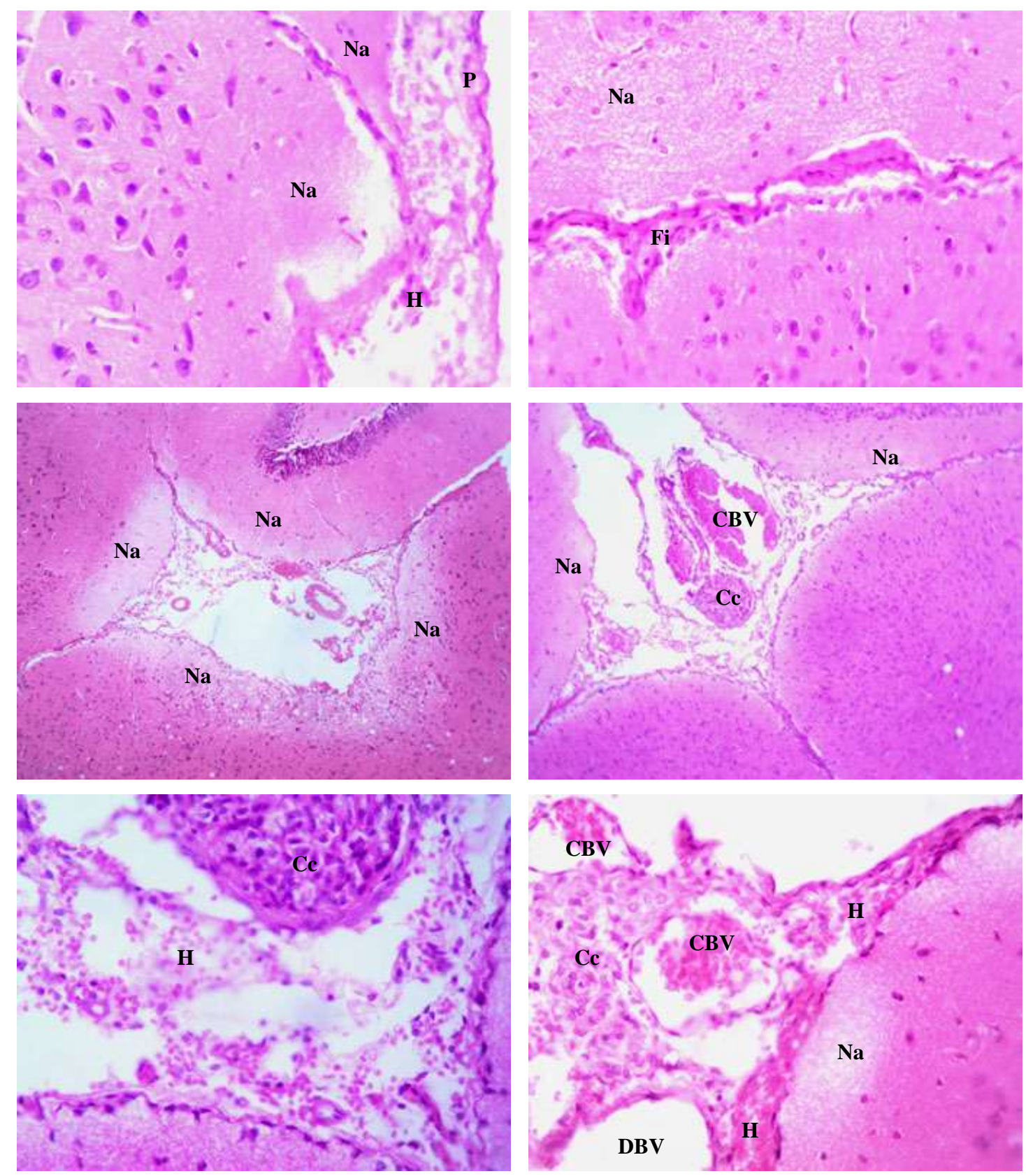

Plate (4) Aluminum Phosphide Group (10 Weeks)

Brain Sections of Albino rats treated with Aluminum Phosphide at 10 weeks. 


\section{5- Diazinon and Vitamin C Group (5 Weeks):}

Photomicrography section of treated rats with Diazinon and Vitamin C in 5 weeks showing: a relatively ameliorative effect on the cerebral tissues, represented by the appearance of normal molecular (Mo), external granular (Eg), internal granular (Ig), external pyramidal (Ep) and internal pyramidal cells (Ip). While the harmful effect of Diazinon still present in the form of shredded pia mater (P) in some areas of treated brains with this combination and also presence of congested blood vessels (CBV) as seen in the (Plate 5). $\mathrm{Hx} \& \mathrm{E} \mathrm{x} 100$ and $\mathrm{x} 400$.
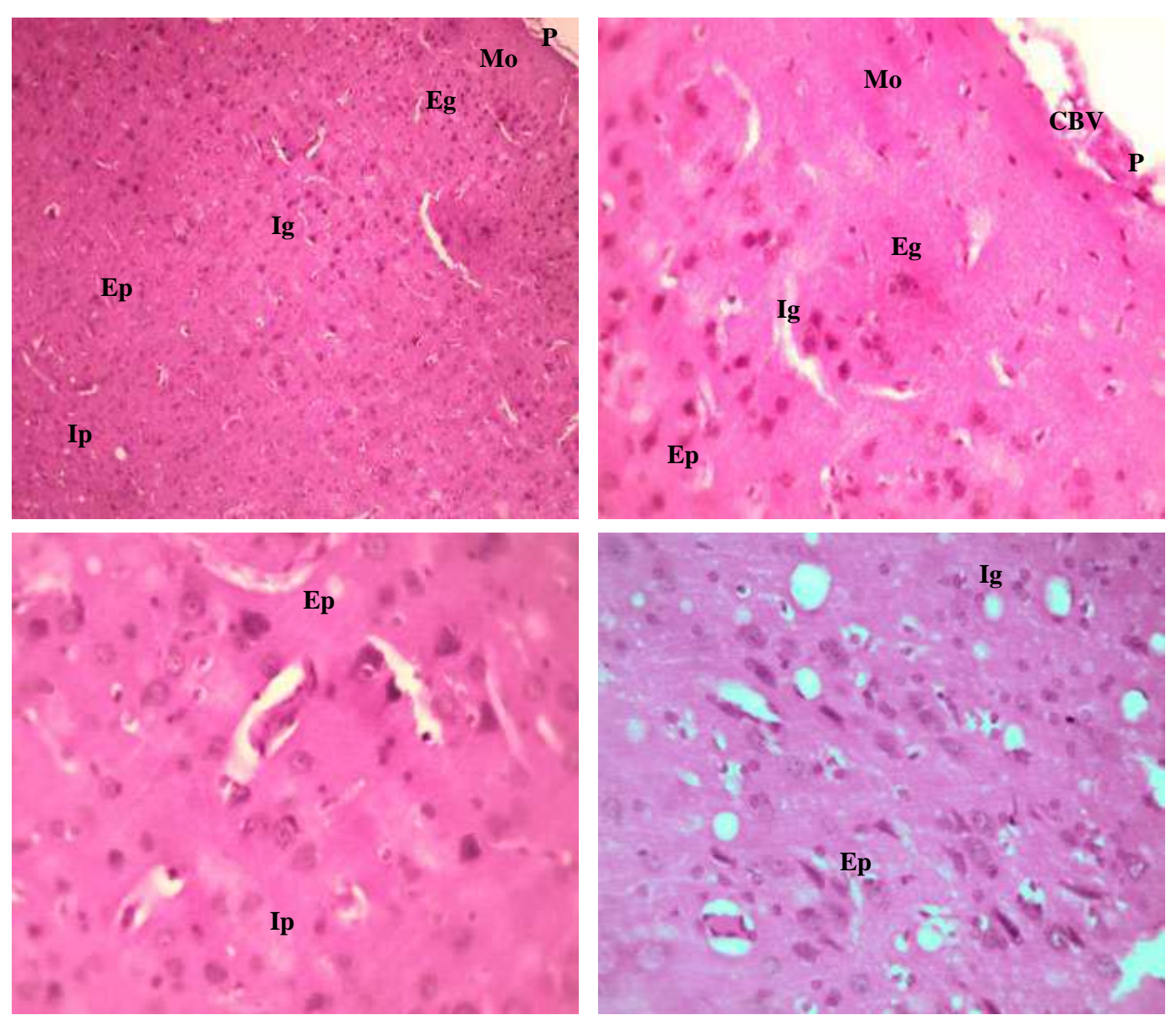

Plate (5) Diazinon and Vitamin C Group (5 Weeks)

Brain Sections of Albino rats protected with Vitamin C against chronic treatment by Diazinon at 5 weeks. 


\section{6- Diazinon and Vitamin C Group (10 Weeks):}

Photomicrography sections of treated rats with Diazinon and vitamin C in 10 weeks show: an improvement in the tissues of brain, represented by the appearance of normal cerebral structure as general, but the damage as a result of treated with Diazinon still found and represented by necrotic areas $(\mathrm{Na})$, separation and shredding of pia mater $(\mathrm{P})$ in some areas, with infiltrated inflammatory cells (Iic) in molecular layer as seen in the (Plate 6). $\mathrm{Hx} \& \mathrm{E} \mathrm{x} 100$ and $\mathrm{x} 400$.
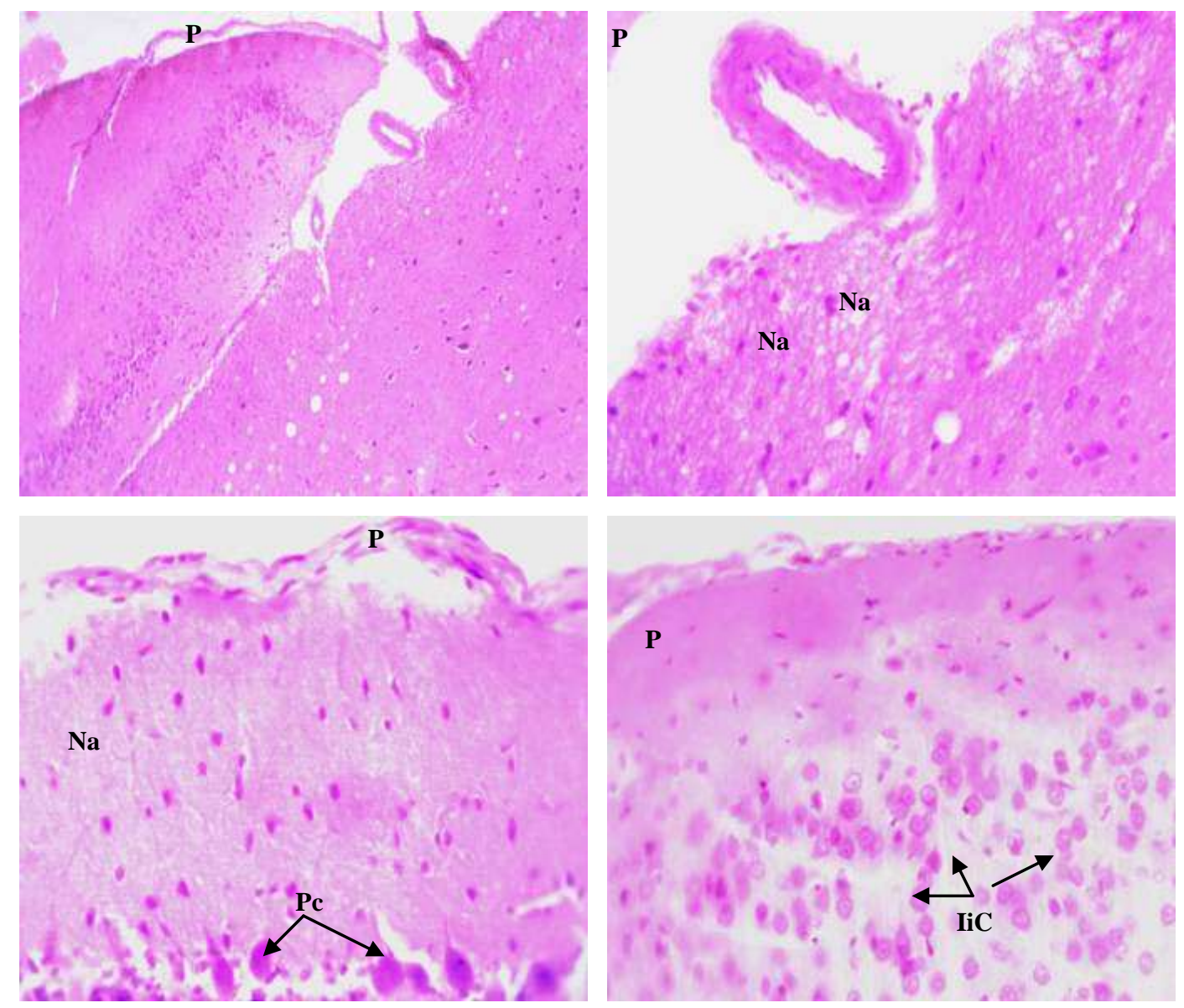

Plate (6) Diazinon and Vitamin C Group (10 Weeks)

Brain Sections of Albino rats protected with Vitamin C against chronic treatment by Diazinon at 10 weeks. 


\section{7- $\quad$ Aluminum Phosphide and Vitamin C Group (5 Weeks):}

Photomicrography sections of treated rats with the combination of vitamin $\mathrm{C}$ and Aluminum Phosphide show a relatively improvement in the tissues of brain, represented by the appearance of normal molecular (Mo), external granular (Eg), internal granular (Ig), external pyramidal (Ep), and internal pyramidal cells (Ip). While the harmful effect of Aluminum still present in the form of infiltrated inflammatory cells (Iic) layer extended from the pia mater to the edges of the external granular, shredding and separation of pia mater $(\mathrm{P})$ in some areas. Necrotic areas $(\mathrm{Na})$, hemorrhage $(\mathrm{H})$, congested blood vessels (CBV) and decrease of the Parkinson cells (Pc) were also seen as in the (Plate 7). Hx\&E x100 and x400.
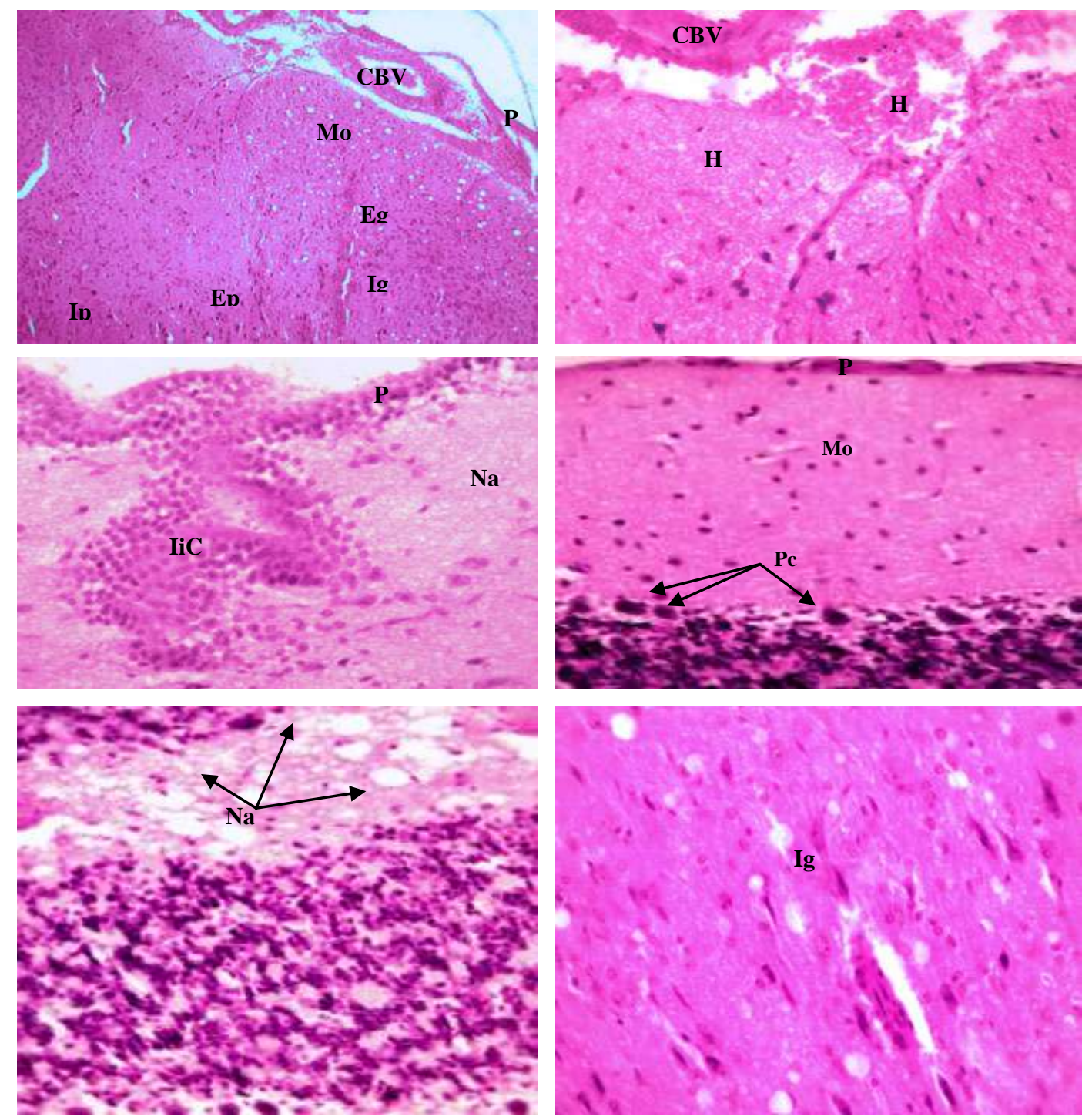

Plate (7) Aluminum Phosphide and Vitamin C Group (5 weeks)

Brain Sections of Albino rats protected with Vitamin C against chronic treatment by Aluminum Phosphide at 5 weeks. 


\section{8- $\quad$ Aluminum Phosphide and Vitamin C Group (10 Weeks):}

The protective effect of vitamin $\mathrm{C}$ in the combination with Aluminum Phosphide (in 10 weeks) in the brain of treated rats show a relatively improvement in the tissues of brain, represented by the appearance of normal molecular layer (Mo), external granular (Eg), internal granular (Ig), external pyramidal (Ep), and internal pyramidal cells (Ip). While the harmful effect of Aluminum still present in the form of fibrosis of the pia mater $(\mathrm{P})$ and separation of it in many areas, congested and dilated blood vessels (CBV) \& (DBV) and the decrease in the number of the Parkinson cells (Pc) were also can seen as in the (Plate 8). HX $\mathrm{x} 100$ and $\mathrm{x} 400$.
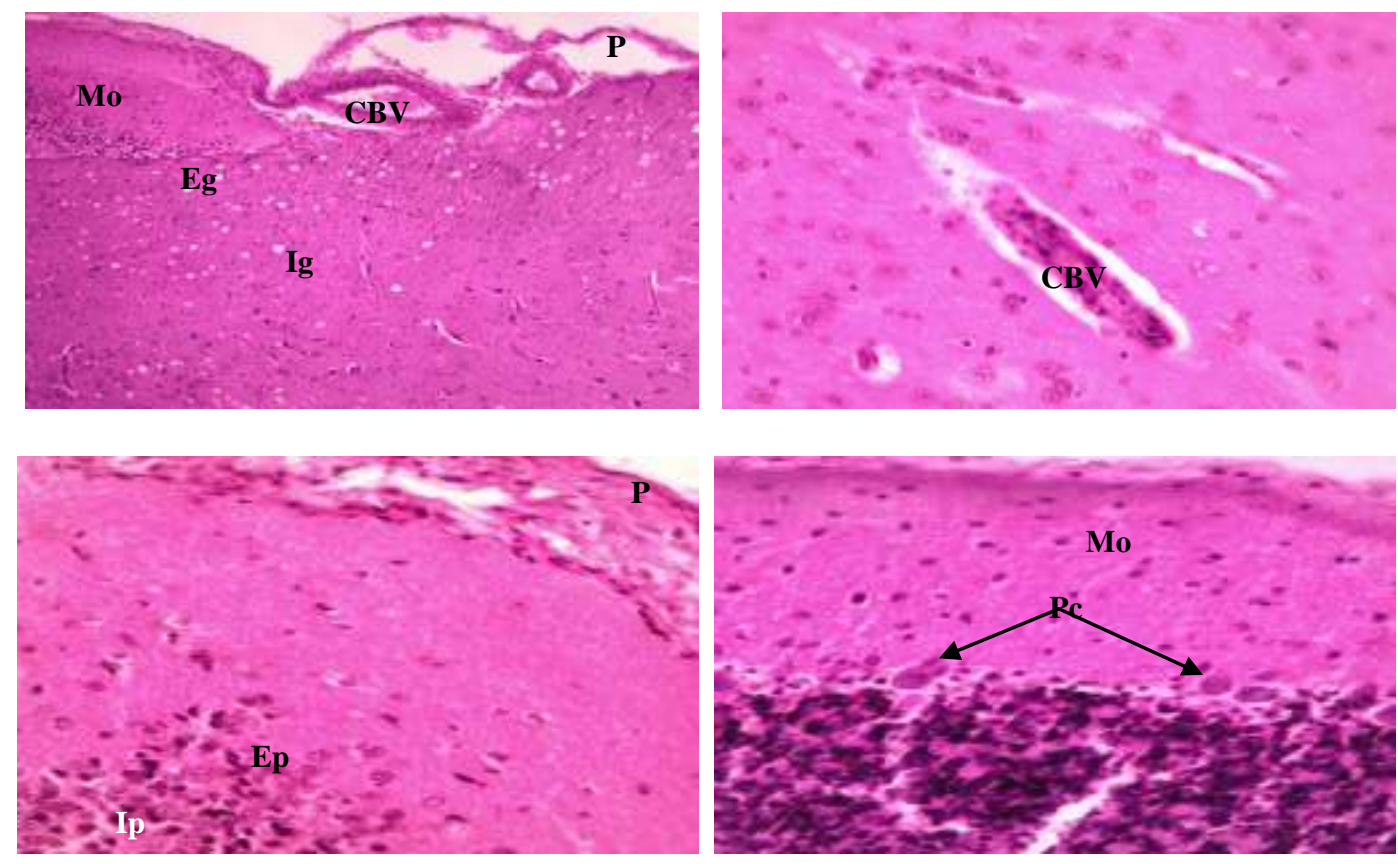

\section{Plate (8) Aluminum Phosphide and Vitamin C Group (10 Weeks)}

Brain Sections of Albino rats protected with Vitamin C against chronic treatment by Aluminum Phosphide at 10 weeks.

\section{DISCUSSION:}

\section{Liver Function tests:}

Alterations in the liver enzymes activity that demonstrate and portray the worthy toxicity which intensified the induced damage caused by the used toxins on the cytoplasmic enzymes as well as its destruction for the intracellular organoids, producing not only cell damage but also liver lesions, fibrosis, exert DNA damage and hepatic carcinoma. Recognizing the assumed restoring and revealing role performed by the used protective Vitamin $\mathrm{C}$ favoring its antioxidant function and induced scavenging for the oxygen free radicals, intercepting the morbidity effects on the important cellular enzymes and biologically active molecules. 
The chronic effect of the chemical synthetic insecticide demonstrated overall significant increase for values of measured (ALT, AST, ALP, glucose and WBCs), significant decrease with total protein, $\mathrm{Hb} \%$ and $\mathrm{RBCs}$. The over dosage of the synthetic materials has deleterious effects on the different organs and may be carcinogenic substances and may causing immunesuppuration, on comparing the side and harmful effect of other natural insecticides. This support the view that naturally an insecticide has less dangerous effects upon the hepatic cells than the synthetic ones, Hassan et al. (2002).

Many enzymes are anchored to cell membranes: one of these enzymes is gammaglutamyl transferase (GGT) which anchored to the biliary canaliculus. Elevation of GGT activity in blood has been considered as indicator of hepatocellular damage, Hanna \& Fayez (1996). Also, serum bilirubin level was increase in rats as a result of treatment with different types of organophosphate pesticides, Gomaa (1995).

The consequence of the used protesting agent (Vitamin C) verses the insulted effects of the used insecticides on the hepatocytes and hepatic parenchyma by acting as free radicals scavenger intercepting these free radicals involved in insecticides intoxification and metabolites degradation by the microsomal enzymes, added for this the pretreatment by the protective agents which exhibited a novel effects on the glutathione status and other enzymatic and non enzymatic antioxidants of the blood, kidney and liver cells.

Many diseases may result in abnormal levels of plasma proteins. Alterations in plasma protein concentration are frequently seen in certain cases of liver diseases and in kidney disease. Margi (1995).

Injury to hepatic tissue resulted in release of cytoplasmic ALT and AST to the blood stream. Accelerated production and release by viable and regenerating hepatocytes may contribute to the serum aminotransferase activity. Injury to the mitochondria results in additional AST release to the blood stream, Meyer et al. (1992).

Diazinon causes changes in liver enzymes and biochemical indices and swelling of mitochondria in hepatocytes (Kalender et al., 2005). Also diazinon causes toxic effects on blood cells, spleen, thymus and lymph nodes of rats (Handy et al., 2002). Meanwhile diazinon causes toxic effects on other organisms (Svoboda et al., 2001).

Ahmed \& Mannaa (2001) studied the protective effect of vitamin $C$ against the toxic action of drinking sodium nitrate contaminated water in adult male rats, the result of this work revealed a protective role of vitamin $\mathrm{C}$ and $\mathrm{E}$ in modulating the toxic effect of sodium nitrate on liver functions as indicated through estimation of serum enzymes AST and ALT.

The effect of high dose of aspirin $(180 \mathrm{mg} / \mathrm{kg} /$ day $)$ on rats leads to a significant elevation in liver enzymes activities, AST and ALT at the end of the experimental period. The administration of a single dose of antioxidants (vitamin $\mathrm{C}, 90 \mathrm{mg} / \mathrm{kg} / \mathrm{day}$ ) to administration of aspirin exerted significant decreased in AST and ALT, El-Batran et al. (2001).

Ahmed \& Mannaa (2001) suggested that vitamin C revealed a protective role in modulating the toxic effect of sodium nitrate on serum total protein concentration. There was no effect on total protein when vitamin $E$ and selenium were intra-peritoneally administrated in rats anesthetized with enflurane, Naziroglu (1999).

Duncan et al., (1994) stated that, albumin is $35-60 \%$ of the total serum protein concentration in animals, it is synthesized by liver. There is direct correlation between albumin turnover and body size, accounts for $75 \%$ of the colloidal osmotic activity of plasma, many constituents of plasma are transported by albumin. 
Moreover, Kamal et al. (1990) reported that long-term exposure of organophosphorus insecticides to a group of 100 Egyptian spraymen showed significantly higher levels of alanine aminotransferase (ALT) and alkaline phosphatase (ALP) but lower levels od cholinesterase (ChE) in serum as compared to control subjects. Concerning the deleterious effects of both (D) and (A) as synthetic and extract insecticides as well as the protective role of Vitamin $\mathrm{C}$ as scavenging agents on the serum Alkaline phosphate (ALP) activity. These alterations in the liver enzymes activity that demonstrate and portray the worthy toxicity which intensified the induced damage caused by the used toxins on the cytoplasmic enzymes as well as its destruction for the intracellular organoids, producing not only cell damage but also liver lesions, fibrosis exert DNA damage and hepatic carcinoma. Recognizing the assumed restoring and revealing role performed by the used protective Vitamin $\mathrm{C}$ favoring its antioxidant function and induced scavenging for the oxygen free radicals, intercepting the morbidity effects on the important cellular enzymes and biologically active molecules. The liver is greatly affected by the chronic treatment with Diazinon (D), Aluminum Phosphide (A), which clarify the protective role of Vitamin C countering the toxicity induced by both (D) and (A) in separated groups by comparing with the control group. This elucidate the induced intensified damage and its lesions on the hepatic cells and parenchyma as a result for the production of oxygen free radicals and detoxification process.

The forgoing speculation resembling that the attributed findings of Murray (1991) and Ceron et al. (1995) they reported that (GGT) activity anchored to the biliary canaliculus and considered that its activity is an indicator for the hepatic cell damage, partial biliary obstruction, in the case of hepatic carcinoma and the liver cirrhosis. Furthermore (Sedki $\boldsymbol{e t}$ al., 2002) concluded that Vitamin $C$ which acts as important antioxidants, enhance the immune system by increasing the lymphocyte contend and production, so provide protection against the toxicity of the environmental pollutants and exposure for insecticides.

Because of the liver is the most important organ in the metabolic processes it is considered as a target organ for the toxic pollutant, vast quantities of data exist on the toxicity of the organophosphorus and the other insecticides mainly on the liver and other organs.

Garg et al. (2005) reported that the dramatic effects concomitance the usage or exposure for the synthetic organophosphorus insecticides and for low extent the naturally performed ones. Furthermore they overt on the other side the brought down of the toxicity, scavenging, restoring and strengthen role of Vitamin $\mathrm{C}$ as most import intracellular antioxidant that performing not only protective effect but also building function for living cells and organoids. The known mode of action for these protective's may be a synergist mechanisms inhibiting lipid peroxidation, mobilization of oxygen free radicals, reduce the risk of free radicals mediated diseases, increase the activity of antioxidant enzymes as well as its role in increasing the liver and blood glutathione, further more it improves the early lipid and collagen accumulation in the liver. Changes in the protein profile elucidate the induced toxicity and liver morbidity. Clarifying the protective role of Vitamin $\mathrm{C}$ countering the toxicity induced by both (D) and (A) in separated groups by comparing with the control group. The liver is the only site for albumin synthesis and because of the liver is a target organ have been implicated in the detoxifications process.

\section{Kidney Function Tests:}

Not only the liver but also the kidney consider as a target organ for insecticides and other pollutants. The results clarify the protective role of Vitamin $\mathrm{C}$ countering the toxicity induced by both (D) and (A) in separated groups by comparing with the control group. 
Creatinine is formed during normal muscle metabolism from the irreversible breakdown of creatine and creatine phosphate. The amount of creatinine present in serum is a function of the total muscle mass present. Muscle cells at a relatively constant rate regardless of diet, age or other factors excrete creatinine. Normally, all plasma creatinine passes through the glomerular membrane and none is reabsorbed. Kidney tubule cells also secrete a small amount of creatinine. Any condition, which impairs glomerular filtration, will result increased serum creatinine levels. Magri (1995).

From the obtained results and observations the used insecticides are frequently elevated serum urea, uric acid and creatinine this may be as a result for the damage implicated in renal function by the multipatent carcinogenic insecticides or its oxidantantioxidant disrupting potential which also may causes hepatorenal lesions or necrosis. This in agreement with the conclusion of Nadeem et al. (2006) who reported that the treatment by either organophosphorus insecticides revealed highly significant increase in the blood urea, uric acid and creatinine in treated animals.

\section{Brain histology:}

The results showed that treatment with Diazinon induced significant $(\mathrm{p}<0.05)$ increases in the level of serum MDA in rat brain. The vitamins $\mathrm{E}$ plus $\mathrm{C}$ combination reduced lipid peroxidation in rat brain (Yilmaz et al., 2012).

Metal phosphides in general and aluminum phosphide in particular are potent insecticides and rodenticides. These are commercially used for protection of crops during storage, as well as during transportation. However, these are highly toxic substances. Their detrimental effects may range from nausea and headache to renal failure and death. It is, therefore, pertinent to ensure their circumspect handling to avoid poisoning episodes (Bumbrah et al., 2012)

Microscopic investigation of the cerebral and cerebellar cortex showed distinct changes due to the effect of celphos. Findings in the cerebral cortex revealed disorganization of the different layers، round shaped neurons with a convex border, degenerated Nissl granules in the cytoplasm and a deeply stained degenerated eccentric nucleus. Findings in the cerebellar cortex included degenerated neurons, the infiltration of round cells into the molecular layer and the disappearance of the processes of Purkinje cells. Degenerate nuclei were surrounded by scavenger cells in the granular layer. The subcortical zone of the brain revealed a paucity of glial cells, degeneration of nerve fibres and the appearance of necrotic patches (Tripathi and Pandey, 2007).

Vitamin $\mathrm{C}$ concentrations in the brain exceed those in blood by 10 -fold. In both tissues 'the vitamin is present primarily in the reduced form, ascorbic acid. We identified the chemical form of vitamin $\mathrm{C}$ that readily crosses the blood-brain barrier, and the mechanism of this process. Ascorbic acid was not able to cross the blood-brain barrier in our studies. In contrast, the oxidized form of vitamin $\mathrm{C}$, dehydroascorbic acid (oxidized ascorbic acid), readily entered the brain and was retained in the brain tissue in the form of ascorbic acid. Transport of dehydroascorbic acid into the brain was inhibited by d-glucose, but not by 1glucose. The facilitative glucose transporter, GLUT1, is expressed on endothelial cells at the blood-brain barrier, and is responsible for glucose entry into the brain. This study provides evidence showing that GLUT1 also transports dehydroascorbic acid into the brain. The findings define the transport of dehydroascorbic acid by GLUT1 as a mechanism by which the brain acquires vitamin $\mathrm{C}$ ، and point to the oxidation of ascorbic acid as a potentially important regulatory step in accumulation of the vitamin by the brain. These results have implications for increasing antioxidant potential in the central nervous system (Agus et al., 1997) 
Ascorbate (vitamin C) is a vital antioxidant molecule in the brain. However, it also has a number of other important functions, participating as a co-factor in several enzyme reactions including catecholamine synthesis, collagen production and regulation of HIF-1 $\alpha$. Ascorbate is transported into the brain and neurons via the Sodium-dependent Vitamin C Transporter-2 (SVCT2), which causes accumulation of ascorbate within cells against a concentration gradient. Dehydroascorbic acid, the oxidized form of ascorbate, is transported via glucose transporters of the GLUT family. Once in cells, it is rapidly reduced to ascorbate. The highest concentrations of ascorbate in the body are found in the brain and neuroendocrine tissues such as adrenal, although the brain is the most difficult organ to deplete of ascorbate. Combined with regional asymmetry in ascorbate distribution within different brain areas 'these facts suggest an important role for ascorbate in the brain. Ascorbate is proposed as a neuromodulator of glutamatergic, dopaminergic, cholinergic and GABAergic transmission and related behaviors. Neurodegenerative diseases typically involve high levels of oxidative stress and thus ascorbate has been posited to have potential therapeutic roles against ischemic stroke, Alzheimer's disease, Parkinson's disease and Huntingdon's disease (Fiona and James, 2009).

\section{CONCLUSION:}

Vitamin C has a protective effect against which harmful effects by Diazinon and Aluminum phosphide insecticides.

\section{REFERENCES}

Abou-Donia, M.B. (1978):"Increased acid phosphatase activity in hens following an oral dose of leplophos. Toxicol. Lett.; 2: 198-203.

Agus, D. B. ; Gambhir, S. S. ; Pardridge, W. M. ; Spielholz, C. ; Baselga, J. ; Vera, J. C. ; and Golde, D. W. ( 1997):" Vitamin C crosses the blood-brain barrier in the oxidized form through the glucose transporters". J Clin Invest. 1; 100(11): 2842-2848.

Ahmed, H.H and Mannaa, F. (2001): "Protective effect of vitamins C and E against the toxication of drinking sodium nitrate contaminated water in adult male rats". J. Egypt. Ger. Soc., 21(A) comparative physiology; 165-185.

Armitage, P. (1974): "Paired student "t" test In: Statistical methods in medical research". Blak well scientific Pub. Oxford, London: 116-120.

Belfield, A. and Goldberg, D. M. (1971): "Colorimetric determination of alkaline phosphatase activity". Enzyme, 12: 561-556.

Ceron, J.J; Panzo, C.C. and Montes, A. (1995): "Toxicological effects in rabbits induced by endosulfan, lidane and methylparthion representing agricultural by products contamination". Bull. Env. Cont. Toxi., 54: 258-265.

Daly, I.W. (1996): "24-month oral Toxicity / Oncogenicity Study in the rat via Dietary Administration: Final Report Huntingdon Life Sciences Project No.: 164 FYF GLP, Unpublished."

Doumas, B.T.; Watson, W.A. and Biggs, H.G. (1971): "Colorimetric determination of serum Albumin". Clin. Chem. Acta., 31: 87-96. 
Dua, R. and Gill, K.D. (2001): "Aluminium phosphide exposure: implications on rat brain lipid peroxidation and antioxidant defence system". Pharmacol Toxicol. 89(6):315-9.

El-Batran, S. A.; Erian, Y. and Arbid, M.S.S. (2001): "Protective effect of some antioxidants against the ulcerative and hepatotoxic effect of aspirin". J. Egypt. Ger. Soc., 28 (A) comparative physiology, $237-248$.

Fiona, E. H. and Jame,s M. M. (2009): "Vitamin C Function in the Brain: Vital Role of the Ascorbate Transporter (SVCT2)” Free Radic Biol Med. 15; 46(6): 719-730.

Fossati, P., Prencipe, L. and Berti, G. (1980): "Colorimetric determination of serum uric acid". Clin. Chem., 26 (2): 227-273.

Garg, M. C.; Chaudhary, D. P. and Bansal, D.D. (2005): "Effect of Vitamin E supplementation on diabetes induced oxidative stress in experimental diabetes in rats". Indian J Exp Biol.; 43 (2): 177-180.

Gomaa, G. M. A. (1995): "Protective effect of phospholipids and some vitamins against insecticides intoxication in male rats". Ph. D. thesis, Dep. Zoo. Fac. Sci., Ain shams Univ.

Guilhermino, L; Soaes, A. M. V. M.; Carvalho, A.P; and Lopes, M. C. (1998): "Effect of cadmium and parathion exposure on haematology and blood biochemistry of adult male rats." Bull. Environ. Contam. Toxicol., 60: 52-59.

Gupta, A. K; Muni, A; Ramhana, A; Dalela, R. C. (1995): "Toxic effect of chlordane and malathion on certain hematological parameters of a freshwater teleost, Notopterus notopterus". J. Environ Biol., 16: 219 - 223.

Gurvinder, S.; Kewal, K.; Tanuj, K.; Madhulika, S. and Gurvinder S. S. (2012): "Phosphide poisoning: A review of literature" Forensic Science International 214: $1-6$

Handy, R.D., Abd-El Samei, H.A. and Bayomy, M.F.F. (2002): "Chronic diazinon exposure: Pathologies of spleen, thymus, blood cells, and lymph nodes are modulated by dietary protein or lipid in the mouse". Toxicology 172:13-34.

Hanna, L. S. and Fayez, V. (1996): "Effect of interaction of hepatic Schistosomiasis mansoni and an organophosphorus poisoning on some biochemical parameters in mice". J. Egypt. Ger. Soc. Zoo1., 19(A): 195 - 220.

Hassan, Y.M.; Zidan, Z.H. Abdel-Daim, Y.A. and Ashoush, I.S. (2002): "Effect of fentrothios and cadmium xenobiotics on some biological systems in male rebbils as indicated to human nutrition Practices". The $1^{\text {st }}$ Conf. Central agric. Pesticide lab. Cairo Egypt, p. 178-180.

Henry, R.J. (1964): "Colorimetric determination of serum total protein". Clin. Chem., Hrper and Row publishers, New York, P. 181.

Henry, R.J. (1974): "Kinetic determination of serum creatinine". Clin. Chem., principles and technics, $2^{\text {nd }}$ Edition, Harper and Row, P. 525.

Hobbiger, F. (1964): " In evaluation of drug activities; pharmacometrics". Eds. Laurence D.R and Bacharach A.L., Pt. 2. Academic Press, London.

Kalender, S.; Kalender, Y.; Ates, A.; Yel, M.; Olcay, E. and Candan, S. (2002): "Protective role Antioxidant vitamin $\mathrm{E}$ and catechin on idarubicin-induced cardiotoxicity in rats"., Braz. J. Med. Biol. Res. 35, pp. 1379 - 1987. 
Kamal, A.A.M., Elgrahy, M.T.; Maklady, F.; Mostafa, M.A. and Massoud, A. (1990): "Serum cholinesterase and liver function among group of organophosphorus pesticides sprayers in Egypt". J. Toxicol. Clinique Exp., 10: 7-8, 427-435.

Margi Sirois, M.S. (1995): "Veterinary clinical laboratory procedures: Mosby's fundamentals of veterinary technology". M. Mosby Inc., Blatimore, Berlin, Boston, Carlessbad, Chicago, London, Madridm Napler, New York, Philadelphia, Sydney, Tokyo, Toronto, $1^{\text {st }}$ Ed.; 2: 214-218.

Matsumura, F. (1985): "Toxicology of Insecticides". $2^{\text {nd }}$ Ed., Plenum Press, New York., p.267-27

Mayne, P.D. (1994): "Protein in plasma and urine In : Clinical Chemistry in diagnosis and treatment" $6^{\text {th }}$ Ed Edward Arnold (London. Boston and Melbourne).

Meyer, D.J.; Coles, E. H. and Rich, L.J. (1992): "Veterinary laboratory medicine: interpretation and Diagnosis". W.B. Saunders Company, Philadelphia, London, Toronto, Montreal, Sydney, Tokyo, $1^{\text {st }}$ Ed.

Mohamed O. S. A.; Adams, S.E.I. and Dirdira, N.I. (1990): "The combines effect of Durshan and Reldan on Nubian goats". Veterinary and Human Toxicology.; 32 (1): 47-48.

Murray, F. (1991):"Get the buzz on bee pollen". Better Nutr, ; 20-21,31.

Nadeem, A.; Anbrin, M.; Nahid, M.; Rabia, A.G. and Zahoor, A.S. (2006): "Immobilization stress causes extra-cellular oxidant-antioxidant imbalance in rats: Restorration by L-NAME and Vitamin E". European Neuropsychopharmacology;16, (4), PP 260-267.

Naziroglu, M. (1999): "Protective role of intraperitoneally administered vitamin E and selenium in rats anesthetized with enflurane". Biological trace element research, 69:3, $199-209$.

Pardue, T.R.; Hansen, E.A.; Barron, R.P. and Chen, J.T. (1970): "Diazinon residues on field-sprayed kale hydroxydiazinon". A New alteration, Products of diazinon.j. Agric., Food Chem. 18 : 405-408.

Patton, C.J. and Crouh, S.R. (1977): "Colorimetric determination of serum urea". Anal. Chem., 94: 464-469.

Reitman, S. and Frankel, S.A. (1957): "A colorimetric method for the determination of serum glutamic oxalacetic and glutamic pyruvic transaminases". J. Clin. Path., 28: 56 .

Roberta, P.; Settimi, L.; Forastiere, F.; Faustini, A. and Zuccaro, P. (2000): "Immunological effects of pesticides exposure". Haelmatologica, 5: 35-42.

Rosalki, S.B., Rau, D. Lehmann and Prentice, M. (1970): "Enzymes chapter 22 quoted from practical clinical biochemistry". William Heinemann Medical Books London PP: 732-735.

Sedki, A. A.; Ismail, A. M.; Abou El-Ella, M. A. abou El-wafa, S.A.; and Abdallah, A. G. (2002): "Performance and immune function of growing rabbits as affected by Vitamin $\mathrm{C}$ and $\mathrm{E}$ through the summer season". Egypt. J. of Agric. Rese., 80 (2):847-864. 
Sethi, P. (2008): "Aluminium induced electrophysiologicalm biochemical and cognitive modifications in the hippocampus of aging rats". Neurotoxicology, 29(6): 10691079.

Sudakin, D.L. (2005): "Occupational exposure to aluminum phosphide and phosphine gas? A suspected case report and review of the literature". Hum Exp Toxicol. 24(1):27-33.

Svoboda, M., Luskova. V., Drastichova. J. and Zlabek, V., (2001): "The effect of Diazinon on hematological indices of common carp (Cyprinus carpio. L)". Acta Vet.Brno., 70, 457-465.

Teitz, N.W. (1986a): "Colorimetric determination of serum bilirubin". Text book of clinical chemistry, W.B. Saunders Co., London, Philadephia, PP. 1389-1390.

Tripathi, S.K. and Pandey S.K.( 2007): "The effect of aluminium phosphide on the human brain: a histological study". Med Sci Law. 47(2):141-6.

Wattersone, A. E. (1999): "Regulating pesticides in the UK: a case study of risk management problems relating to the oragnophosphat diazinon". Toxicol. Lett., 107: 241-248.

WHO (World Health Organization), (1988): "Phosphine and selected metal phosphides", vol.73. Environmental Health Critaria, Geneva, Switzerland, p. 104-110.

WHO. Environmental Health Criteria (1998): "Diazinon; International Programme on Chemical Safety". World Health Organization: Geneva, Switzerland, p. 3-5.

Yilmaz, N.; Yilmaz, M. and Altuntas, I. (2012): "Diazinon-induced brain toxicity and protection by vitamins E plus C". Toxicol Ind Health. 28(1):51-7.

$$
\begin{aligned}
& \text { الاور الوقائى لفيتامين "ج" ضد تغيرات فسيولوجية وهستولوجية فى الجرذان }
\end{aligned}
$$

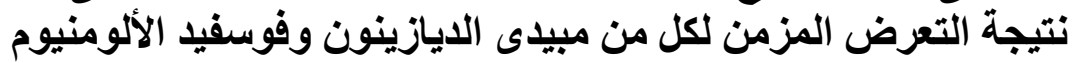

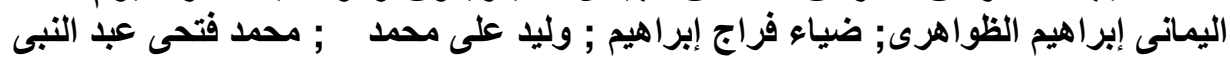

$$
\begin{aligned}
& \text { قسم علم الحيوان- كلية العلوم (بنين) - جامعة الأزهر. }
\end{aligned}
$$

يتم استخدام المبيدات الحشرية ضدن العديد من المواد الكيميائية التى تو اجه الإنسان بومبا والتى تضاف للبيئة

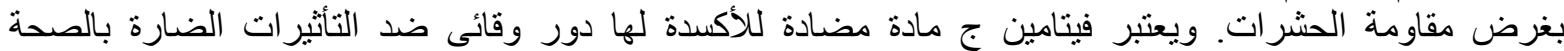

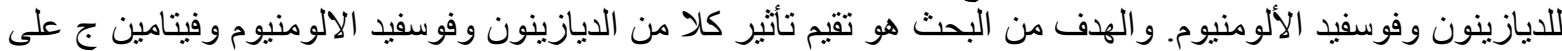

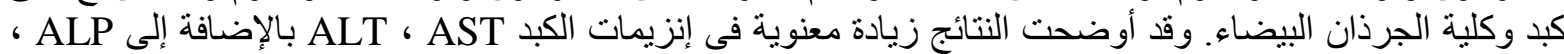

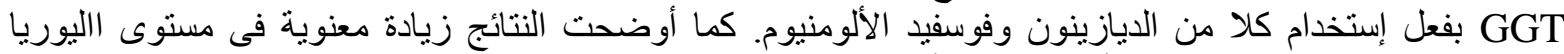

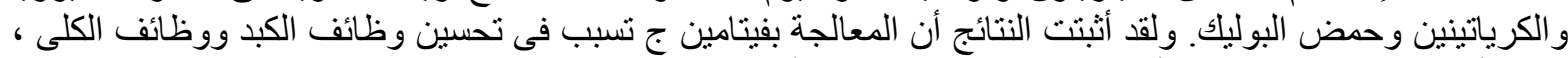

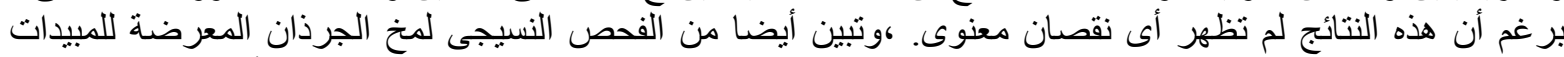

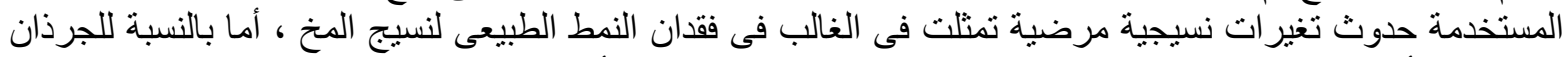

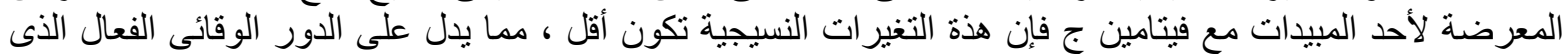
تقوم بـه مثل هذه المركبات.

والإستنتاج: بستطيع فيتامين ج أن يحسن أى تغير ات ضارة للايازينون وفوسفيد الألومنيوم على كلا من الكبد والكلى 
